\title{
Gradhiva
}

GRADHIV

Revue d'anthropologie et d'histoire des arts

9 | 2009

Arts de l'enfance, enfances de l'art

\section{Des jeux aux mythes :le parcours ethnographique de Marcel Griaule}

From games to myths: the ethnographic career of Marcel Griaule

\section{Éric Jolly}

\section{(2) OpenEdition}

\section{Journals}

Édition électronique

URL : http://journals.openedition.org/gradhiva/1441

DOI : 10.4000/gradhiva.1441

ISSN : 1760-849X

Éditeur

Musée du quai Branly Jacques Chirac

\section{Édition imprimée}

Date de publication : 2 septembre 2009

Pagination : 164-187

ISBN : 978-2-35744-009-8

ISSN : 0764-8928

\section{Référence électronique}

Éric Jolly, «Des jeux aux mythes :le parcours ethnographique de Marcel Griaule », Gradhiva [En ligne], 9 | 2009, mis en ligne le 02 septembre 2012, consulté le 01 mai 2019. URL : http://

journals.openedition.org/gradhiva/1441 ; DOI : 10.4000/gradhiva.1441

(c) musée du quai Branly 







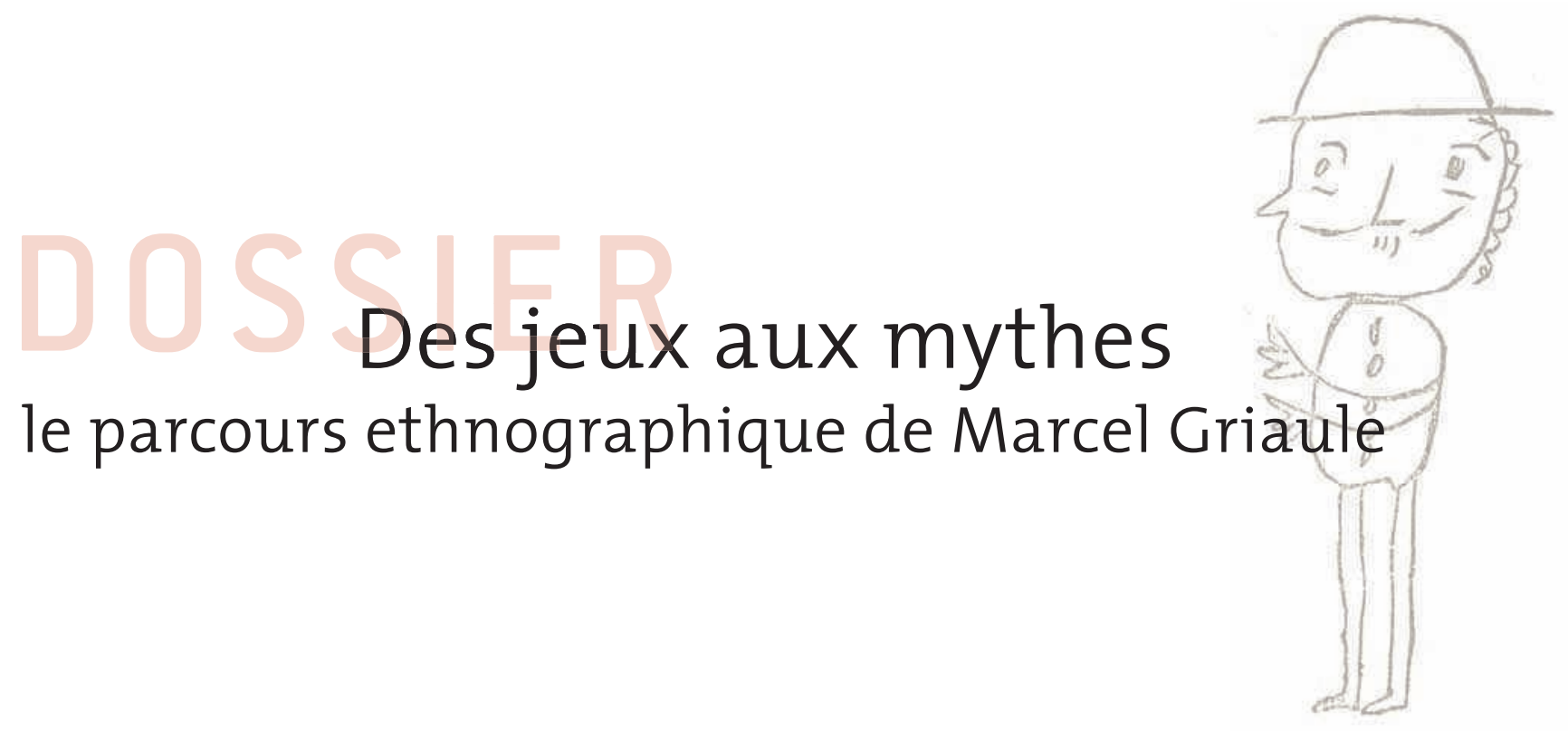

\section{Éric Jolly}

Fig. 1 Poupées toucouleur (à gauche) et mande (à droite), collectées en 1931 dans la région de Kayes, Mali (mission Dakar-Djibouti, musée du quai Branly).
$\mathrm{Au}$ cours de ses premières enquêtes en Éthiopie et au Soudan français, l'ethnologue Marcel Griaule travaille essentiellement avec les enfants, en particulier sur leurs jeux et leurs dessins. À partir de 1946, en revanche, à l'intérieur comme à l'extérieur du pays dogon, il n'interroge plus que des adultes, concentrant ses recherches sur les mythes, les signes, les secrets et les connaissances supérieures détenus selon lui par les vieillards ou les initiés. Cet article analyse les raisons, la genèse et les conséquences de ce saut thématique, en postulant une rupture plus méthodologique que théorique. En effet, Griaule porte un regard relativement stable sur les sociétés africaines qu'il étudie, même s'il modifie sa façon de les aborder. Jusqu'aux années 1950, il continue de concevoir les cultures des enfants et des adultes comme deux mondes contigus, successifs et complémentaires. À ses yeux, le monde liminaire des enfants, libre, ouvert et ludique, est censé s'opposer, mais également préparer au monde clos, figé et religieux des générations supérieures. Sa vision des productions enfantines et sa perception des savoirs graphiques ou mythologiques des vieillards ne sont donc pas dissociables : elles se nourrissent mutuellement, par un effet de miroir. Combinées à l'idée qu'il existe une stratification du savoir, ces représentations expliquent par ailleurs le parcours de Griaule, ou le justifient a posteriori. Ou'il s'agisse de pénétrer progressivement jusqu'au cœur de la société ou de se hisser jusqu'à sa clé de voûte mythologique et symbolique, l'étude des enfants et de leurs jeux est bien, selon cette conception, la porte d'entrée idéale.

Prélude ethnographique : l'enfant et ses productions élémentaires (1928-1939]

Entre 1928 et 1939, lors de ses cinq premières missions, Griaule porte un intérêt particulier, mais non exclusif, aux jeux et aux dessins des enfants africains. Du Soudan français à l'Éthiopie et du Cameroun au Tchad, son équipe collecte près de 500 jouets et mène des enquêtes systématiques auprès de jeunes informateurs, avec à la clé onze publications et plusieurs exposés sur les productions enfantines, dont trois articles, trois livres et une conférence radiophonique signés 
Griaule. Pourquoi cette attention accordée d'emblée aux activités ludiques? Souvent jugée "mineure ", la culture enfantine n'en est pas moins "première » aux yeux de Griaule et des chercheurs de cette époque.

\section{GENĖSE d'UN INTÉRÊT ETHNOLOGIQUE POUR LES PRODUCTIONS ENFANTINES}

À la fin des années 1920, en France, les ethnographes se rapprochent de l'avantgarde artistique ou littéraire en raison de plusieurs idées, positions ou inclinations communes : le rejet de l'esthétisme, le refus de toute hiérarchie entre les différents éléments constitutifs d'une culture, la contestation de la primauté de l'Occident, et un goût marqué pour les arts et les rites des sociétés exotiques. Influencés par le primitivisme, chercheurs et artistes partagent également une vision similaire des jeux ou des dessins enfantins ${ }^{1}$ : ces productions, comme celles des sociétés dites "primitives", leur paraissent témoigner d'une culture originelle plus ou moins préservée, heureusement indemne de toute contamination (par l'école ou par l'Occident). Selon un postulat ancien repris tant par Marcel Mauss que par Griaule (1934:12-14; $1935: 5 ; 1938$ : 1-2), les jeux conserveraient d'ailleurs les traces de rites ou de mythes aujourd'hui disparus.

Ces visions convergentes et cette curiosité partagée s'expriment en particulier dans les colonnes de l'éphémère revue Documents, qui, conformément à son sous-titre, associe chaque mois «archéologie, beaux-arts, ethnographie». De 1929 à 1930, les surréalistes dissidents y côtoient les jeunes ethnographes récemment formés par l'Institut d'ethnologie. Dès son retour d'Éthiopie, Griaule collabore activement à Documents : il remplace Michel Leiris au secrétariat de rédaction et rédige plusieurs articles, dont sa première note sur les "jeux abyssins». Il y décrit brièvement, dans la rubrique "Métamorphose " (Griaule 1929: 332), des imitations d'animaux destinées à amuser ou effrayer les jeunes enfants éthiopiens. Un an plus tard, en raison d'un simple jeu d'homonymie, plusieurs photographies de jouets amérindiens accompagnent son article sur les statuettes africaines improprement appelées « Joujou » ou "Djoudjou » (Griaule 1930 : 367-368). Conforme à l'esprit de la revue, ce rapprochement inattendu n'en confirme pas moins l'intérêt porté aux jeux d'enfants, a fortiori s'ils viennent de pays lointains. Dans le numéro suivant, les dessins enfantins sont de nouveau à l'honneur : une vingtaine de graffiti éthiopiens relevés par Griaule illustrent sur une double page un texte du secrétaire général de Documents, Georges Bataille (1930: 394-395). Intitulé «L'art primitif", cet article discute en partie, sans les réfuter, les liens formels établis par Georges-Henry Luquet (1930) entre les dessins des enfants et l'art des hommes préhistoriques ou des sociétés «primitives ». Et les quelques lignes de commentaires sur les graffiti éthiopiens appuient cette affirmation implicite d'un "art premier " enfantin, libre de tout carcan et vierge de toute influence. Exécutés à l'insu des adultes, ces dessins areligieux sont en effet présentés par

1. Sur l'intérêt des avant-gardes, dans l'entre-deux-guerres, pour un art authentique et alternatif propre à la fois aux «primitifs » et aux enfants, voir l'article de Philippe Sabot (2003: 117-119).

2. Bataille $1930: 396-397$, note 3 .

3. Voir l'introduction de Daniel Fabre.
Bataille (1930 : 396), sur les indications de Griaule, comme de «bizarres élucubrations", synonymes d'espièglerie, de transgression ludique et de liberté expressive. En note ${ }^{2}$, Bataille ajoute que les enfants éthiopiens semblent jouer avec les formes comme ils joueraient avec les mots, en leur donnant plusieurs sens. Cette hypothèse est loin d'être anodine : elle traduit l'idée récurrente selon laquelle les jeux s'inscrivent dans une "esthétique générale” " en combinant, par souci de plaisir, des expériences créatrices très diverses (d'ordre intellectuel, graphique, oral, manuel, corporel, musical, etc.). 
La stylisation demande tant d'additions, de retranchements, de déplacements de valeurs que le joueur [de ficelle] en est un instant bousculé en cette partie élevée de son esprit, qui n'est ni religieuse, ni technique, ni morale, mais réservée à la spéculation désintéressée, aux rêves irréalisables et, pour tout dire, à l'activité esthétique (ibid. : 11).

Pour expliquer l'intérêt initial de Griaule pour les jeux, il faut encore mentionner la probable influence de son mentor scientifique, le linguiste éthiopisant Marcel Cohen. Membre de l'école de sociologie française et cofondateur de l'Institut d'ethnologie, Cohen avait publié dès 1911, à partir de ses matériaux de terrain, une longue note descriptive et comparative sur les « Jeux abyssins ». Sur le plan ethnographique, les premiers travaux de Griaule s'inscrivent donc dans la continuité des recherches entreprises par son professeur d'amharique.

\section{VERS UNE ENCYCLOPÉDIE DES JEUX AFRICAINS}

Si Griaule commence sa carrière d'ethnologue au musée d'Ethnographie du Trocadéro, avec le soutien de Paul Rivet et Georges-Henri Rivière, ses liens avec l'institution pèsent davantage sur ses options méthodologiques que sur ses choix thématiques. La double perspective muséographique et monographique défendue par Mauss, Rivet et Rivière le pousse tout au plus à considérer les jouets comme des témoignages culturels aussi dignes d'étude qu'un outil ou un masque d'adulte, mais elle l'oblige surtout à effectuer conjointement des collectes systématiques d'objets et des enquêtes de terrain méthodiques sur tous les sujets. Conjuguée à son intérêt particulier pour les jeux, cette méthode se traduit avant guerre par un achat massif de jouets pour le compte du musée d'Ethnographie, et par la constitution d'une documentation complète sur les activités ludiques des enfants, grâce à une combinaison de fiches descriptives et d'enregistrements photographiques, phonographiques ou plus rarement filmiques ${ }^{6}$.

Sur le terrain, la priorité est de tout saisir, inventorier et archiver - des rites les plus complexes aux jeux les plus simples7 -, en visant l'exhaustivité à l'échelle de chaque société ou de chaque thème abordé. Dans ses rapports publiés, Griaule insiste par conséquent sur les résultats quantitatifs de chacune des missions collectives dont il a la direction, en particulier dans le domaine des jeux. Il annonce par exemple avoir recueilli, entre mai 1931 et novembre 1932, des informations complètes sur trois cents jeux dogon et une centaine de jeux éthiopiens, contre deux cent cinquante lors de sa précédente expédition (Griaule 1932 : 115; 1932b : 231). En revanche, à l'exception parfois des poupées, les jouets collectés ne font pas l'objet d'un décompte séparé. Ils représentent environ 250 des 3600 objets rapportés par Dakar-Djibouti (1931-1933), une vingtaine sur trois cents pour Sahara-Soudan (1935) et une soixantaine sur huit cents pour Sahara-Cameroun (1936-1937), sans compter les objets de fouilles et leurs nombreux jouets en terre (Lebeuf 1945 : 133). Enfin, la mission de 1938-1939 (Lebaudy-Griaule ou Niger-Lac Iro) dépose au musée de l'Homme une sélection de cent quarante objets, dont une immense majorité de jouets. À l'instar de l'enquête ethnographique, cette collecte muséographique se plie à l'exigence d'exhaustivité, que ce soit par la constitution de séries complètes, avec une importante collection de poupées ${ }^{8}$, ou au contraire à travers l'achat d'échantillons très divers, choisis indépendamment de leur beauté, de leur fragilité ou de leur complexité. Sur son trajet, chaque mission Griaule "rafle " ainsi, avec méthode et boulimie, tous les jouets 
qu'elle peut acquérir, de la montre en capsule de bouteille à l'automobile en tiges de mil, dans la perspective d'un inventaire complet de la culture matérielle des enfants9. Les témoignages des membres de la mission Dakar-Djibouti sont à cet égard éloquents, surtout en début de parcours ${ }^{10}$ :

Rafle, comme dans les autres villages, de tout ce qu'on peut trouver en fait de costumes de danse, objets usuels, jouets d'enfant, etc. [28 août 1931] (Leiris 1981 : 77).

Cette volonté d'exhaustivité et d'inventaire se traduit également, en amont du terrain, par l'établissement d'un questionnaire sur les jeux ${ }^{11}$, et, en aval, par la rédaction de deux monographies sur les jeux éthiopiens et dogon, publiées par Griaule en 1935 et 1938. Chacun de ces ouvrages s'apparente à un catalogue descriptif de quatre à cinq cents jeux, avec leurs variantes locales ou régionales. Ils se présentent comme une succession de notes indépendantes reproduisant le contenu des fiches de terrain, selon un procédé clairement revendiqué par Griaule (1935: 6). L'objectif est d'offrir au lecteur des documents ethnographiques "bruts», complets et limpides, en s'interdisant les artifices littéraires, en se méfiant des correspondances mythologiques et en se gardant de toute

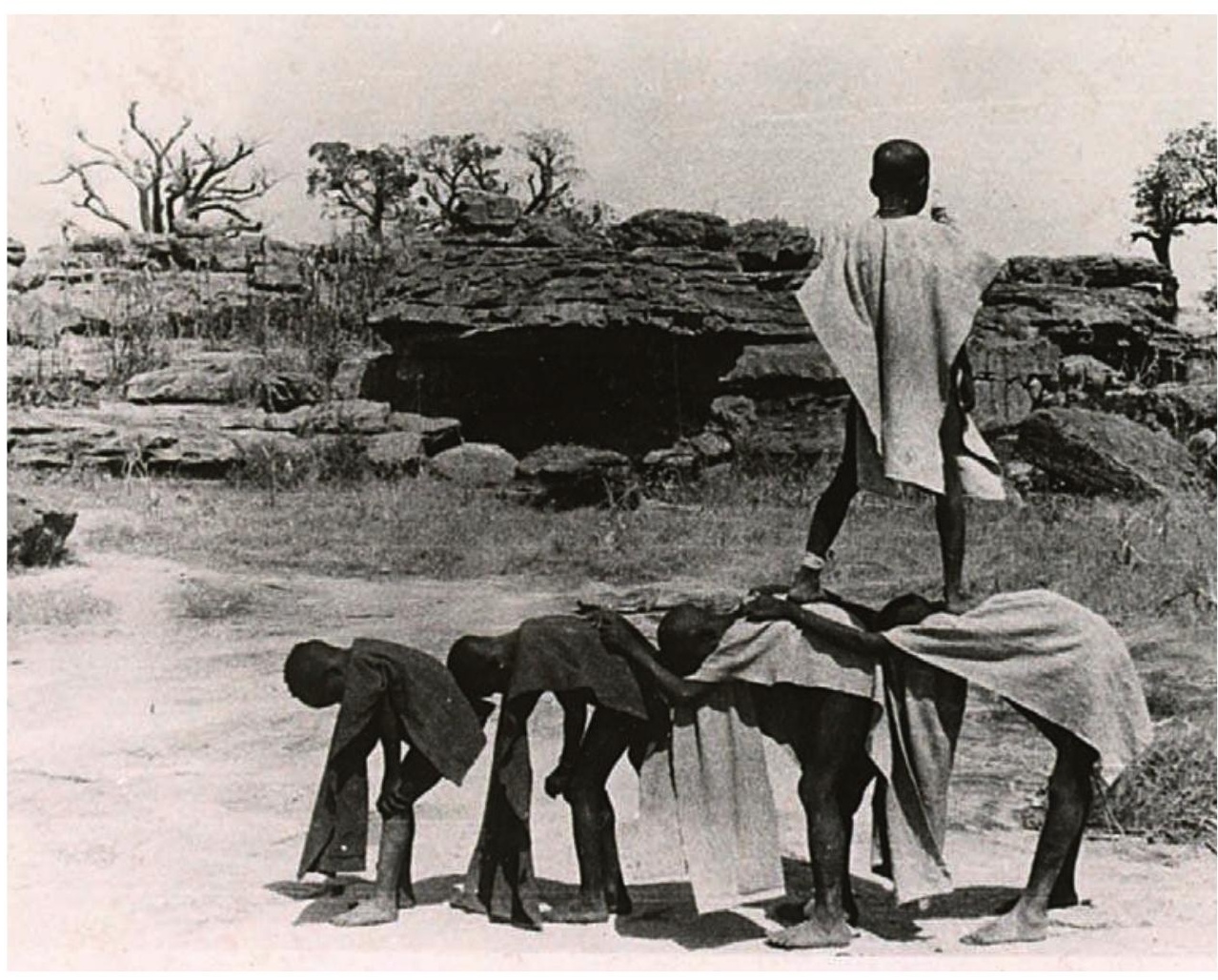
systématisation ou synthèse (ibid. : 5). Pour chaque jeu, Griaule propose une courte description de son déroulement ou du processus de fabrication du jouet, avant de préciser d'un mot sa localisation ainsi que le sexe du joueur. Des dessins réalisés à partir de photographies complètent et explicitent ces descriptions en reproduisant, soit le jouet, soit les différentes séquences ou figures du jeu. Transcrits et traduits, les chants des enfants sont également joints à ce descriptif, tandis que des notes de bas de page mentionnent, le cas échéant, les numéros d'inventaire des jouets déposés au musée du Trocadéro. Enfin, le texte renvoie éventuellement aux planches photographiques placées en fin d'ouvrage. En croisant texte, dessins, photographies, enregistrements et objets, Griaule vise la restitution de la réalité, ou au besoin sa reconstitution. Le procédé est conforme aux directives méthodologiques des années 1930, mais il est également lié au sujet traité. Les jeux des enfants se prêtent d'autant mieux au catalogage et aux descriptions réalistes qu'ils sont euxmêmes jugés «terre à terre" par Griaule, en raison de leur proximité avec la vie courante et de leur indépendance présumée par rapport aux mythes, aux secrets et à la religion des adultes : " les jeux ne doivent rien à la cosmogonie. Ils sont terre à terre, sans jeu de mots » (Griaule $1938: 4$ ).

Fig. 3 Reconstitution d'un jeu d'enfants à Sangha, Mali (photographie de Marcel Griaule, novembre 1931, mission Dakar-Djibouti. Musée du quai Branly).

\section{- -}

9. Pour Griaule, toute production spécifiquement enfantine relève en effet de la catégorie «jeu », depuis le dessin jusqu'au piège à souris ou au mirliton.

10. Voir également Lutten 1934 : 79.

11. Griaule a établi ce questionnaire avant sa mission de 1928 à partir des travaux antérieurs de Marcel Cohen, mais aussi de ses premières enquêtes menées auprès d'Éthiopiens résidant à Paris (Griaule 1935 : 1). 


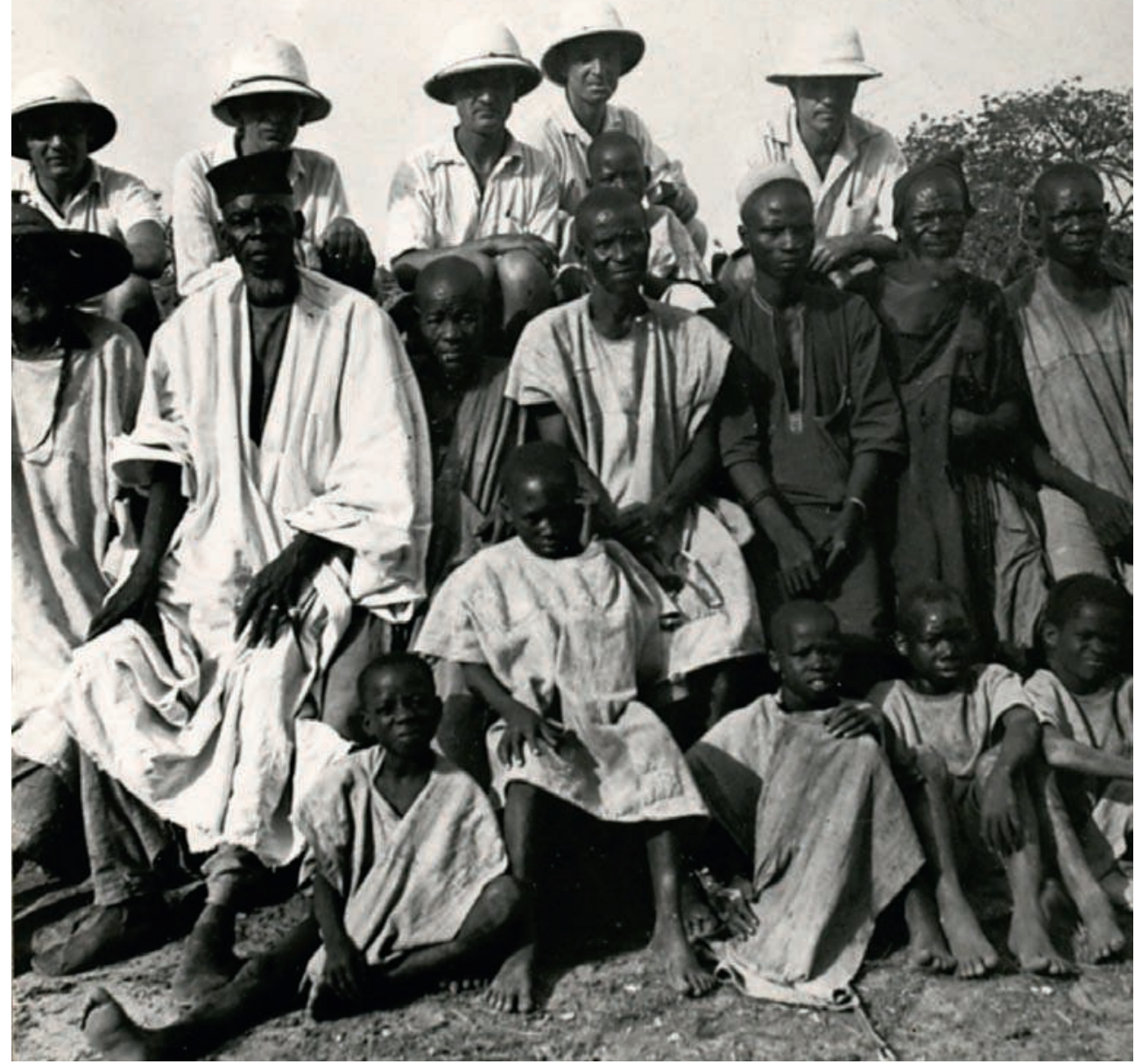

L'inventaire des jeux d'enfants ne devait pas s'arrêter aux Dogon et auxÉthiopiens. Àl'issue de sa cinquième mission, Griaule envisage, avec ses collaborateurs, "l'établissement d'une encyclopédie des jeux africains" grâce à de nouvelles publications sur les populations kouroumba, fali et sara (Griaule 1943: 683). Mais, en dépit de quelques articles ultérieurs de Jean-Paul Lebeuf ou de Griaule $^{12}$, le projet finit par avorter, en raison de l'abandon, après guerre, des objectifs initiaux d'exhaustivité ou d'encyclopédisme.

Fig. 4 Les membres de la mission Dakar-Djibouti et leurs informateurs ou interprètes dogon à Sangha, Mali, en septembre 1931. Au premier rang, de gauche à droite, les jeunes Abara, Amadigné et Iréko (musée du quai Branly).

\section{- $\bullet$}

12. Cf. Lebeuf 1941, 1983, 1991 ; Griaule et Ligers 1955. Portant sur les jeux fali, kotoko ou goula, les trois articles de Jean-Paul Lebeuf, même les plus tardifs, se fondent sur les notes de terrain prises lors des missions Griaule de 1936-1937 et 1938-1939, au Cameroun et au Tchad. En revanche, l'article sur un jeu bozo, cosigné par Griaule, se nourrit en partie d'informations recueillies au Soudan français en 1952 (registre de mai 1952 : 470, fonds Marcel-Griaule).

\section{INFORMATEURS AVANT GUERRE : L'ENFANT INNOCENT VERSUS L'ADULTE COUPABLE}

Dans le contexte des expéditions itinérantes dirigées par Griaule, l'ampleur et l'efficacité des recherches sur les jeux s'expliquent également par l'âge des spécialistes sollicités. Comparés aux adultes, les enfants, ou du moins les garçons et plus encore les écoliers, se révèlent de merveilleux collaborateurs pour des enquêtes ou des collectes rapides, en raison à la fois de leur disponibilité, de leur vitalité, de leurs rudiments de français et de leur absence de réticence à être interrogés, photographiés ou dépossédés de leurs productions éphémères. En d'autres termes, ils se prêtent plus facilement au "jeu " et aux exigences des enquêteurs, en se transformant à volonté en informateurs, interprètes, porteurs, producteurs de jouets, déterreurs de trésors, collecteurs d'insectes, spécimens anthropologiques (pour les prises de vues anthropométriques), ou enfin en acteurs talentueux (pour reconstituer tel ou tel jeu dans une ambiance doublement ludique). Comme le reconnaît Leiris au début de la mission DakarDjibouti, «il est beaucoup plus intéressant de travailler avec les enfants qu'avec les adultes" (Leiris $1981: 63$ ).

Certains écoliers dûment rétribués deviennent d'ailleurs des auxiliaires d'enquête essentiels, bien au-delà de la simple thématique du jeu. Complices et complaisants, ces jeunes élèves sont soumis à des séances de travail intensives et répétées. C'est ainsi qu'à Kita et à Bamako, en 1931, ils doivent se soumettre aux questions successives et insistantes d'au moins trois membres de l'expédition Dakar-Djibouti. Tout en enregistrant leurs chants et en notant leurs contes, Jean Mouchet complète auprès d'eux ses informations linguistiques, tandis que Leiris et éventuellement Griaule, à une table voisine, les interrogent sur les sociétés enfantines et sur les rites de circoncision ou d'excision. 
Arrivés au convoi [...], nous trouvons le wagon jonché d'élèves [...] que Mouchet a laissé se reposer pendant qu'il déjeunait et qui dorment en attendant de reprendre l'enquête [Bamako, 14 août]. [...] Travail fou avec les circoncis. [...] Trois enquêteurs fonctionnent simultanément et à jet continu : Mouchet à une table avec deux des enfants, moi à une autre table avec les deux autres enfants, Griaule n'importe où [Bamako, 15 août] (ibid. : 69-70).

Le rendement est tel que Griaule évoque, dans sa correspondance ${ }^{13}$, une "enquête énorme", fondée sur une "méthode "industrielle" ». À Kati, en attendant le départ imminent du train et malgré une pluie diluvienne, Griaule, penché à la portière, continue à interroger des enfants trempés perchés sur les marches du wagon ${ }^{14}$. Bien entendu, il ne faut voir, dans les scènes précédentes, ni exploitation ni obséquiosité : les écoliers se plient aux conditions de travail avec enthousiasme, insouciance, candeur, fierté et bonne humeur, en étant libres de déguerpir à tout moment. Attachés à ces curieux et prestigieux étrangers, voire collés à eux, les enfants ont en fait beaucoup de mal à s'en détacher, quel que soit le lieu de l'enquête, et pour toutes les missions d'avant guerre. Ils invitent les ethnographes à leurs festivités ou dans leur famille, les accompagnent partout, s'incrustent ou se réfugient dans leur campement, fabriquent des jouets à leur intention et, pour certains, rêvent ouvertement de les suivre ${ }^{15}$.

Certains y parviendront d'ailleurs, à l'insu de leurs parents, comme ce jeune informateur bamakois de Dakar-Djibouti, Mamadou Keyta, âgé de treize ans (voir fig. 6). Il est vrai que Griaule avait lui-même proposé de l'engager et de l'emmener comme interprète, avec l'intention d'en faire un "grand ethnographe " pour la suite de l'expédition ${ }^{16}$, en pariant sans doute sur sa malléabilité. Mais, en dépit de la formation accélérée inculquée par Mouchet et contrairement aux satisfecit officiels ${ }^{17}$, Mamadou Keyta se révèle un piètre enquêteur : sur l'ensemble du tronçon entre Bamako et Yaoundé, il ne rédige que trois fiches avant d'être finalement «licencié ", alors que Griaule songeait initialement à le ramener en France ${ }^{18}$. Un tel échec était pourtant prévisible. En se comportant en enfants et en étant traités comme tels, les écoliers se révèlent d'agréables informateurs, mais ils n'ont pas l'autorité nécessaire pour jouer les enquêteurs selon les méthodes prônées par Griaule, en se transformant en ethnographes paternels (vis-à-vis des jeunes de leur âge) ou en détectives suspicieux (vis-àvis des adultes).

Dans les années 1930, les membres des missions Griaule ont en effet des rapports très différents avec leurs informateurs adultes. S'ils se plaisent en compagnie des écoliers, en nouant avec eux une relation de proximité et de complicité teintée de paternalisme, ils adoptent, face à leurs interlocuteurs adultes, un comportement plutôt distant, ferme, inquisiteur et combatif, en étant vite agacés par leurs silences, leurs contradictions ou leurs pirouettes verbales. Cela n'a rien de surprenant au regard de leurs méthodes d'enquête et du contexte colonial, mais cette attitude presque opposée est aussi légitimée, et donc accentuée, par la distinction opérée par Griaule entre l'univers des enfants et celui des adultes, en particulier sur le plan du savoir (et des relations à ce savoir). Aux yeux de Griaule, l'enfant n'a rien à cacher et peut donc tout dire, librement et ingénument. L'adulte, à l'inverse, est le dépositaire présumé d'un savoir initiatique - secret et supérieur - qu'il masque à l'étranger pour lui interdire l'accès aux connaissances clés de sa société. D'emblée, tout informateur adulte est donc soupçonné de dissimulation, de mensonge, de résistance obstinée ou de

\section{-}

13. Lettre de Griaule à G.-H. Rivière, 18 août 1931, Bamako (archives du musée de l'Homme, 2AM1M2c).

14. Lettre de $M^{\text {me }}$ Griaule à G.-H. Rivière, 18 août 1931 (ibid.).

15. Leiris $1981: 47,71,74,112$, 123, 126, 173 ; Gordon 1935, II : 1, III : 1, VIII : 2 .

16. Leiris 1981 : 75, 84-85, 183. La nouvelle semble suffisamment surprenante pour être reprise par certains journaux français de l'époque : «À Bamako, M. Griaule a engagé comme interprète un négrillon de treize ans qui, paraît-il, fait preuve d'une rare intelligence » (Fortolis 1931).

17. Dans un rapport de la mission Dakar-Djibouti, Griaule note que l'élève «qui les a accompagnés pendant plusieurs mois s'est révélé capable d'effectuer seul de petites enquêtes ethnographiques » (Griaule 1932 : 119).

18. Lettre de Leiris à sa mère, 18 février 1932 (Leiris 1996 : 355, note). 
-

19. Sur ces multiples métaphores militaires ou judiciaires appliquées à l'enquête ethnographique, voir : Griaule 1943: 58, 60, 74; 1952 : $545 ; 1957$ : 19, 59; Jamin 1982 : 87-89.

20. On peut toutefois se demander si la théorisation d'un tel épisode ne sous-estime pas la volonté d'Abara de se mettre totalement au service de Griaule, auquel il est très attaché. Dans un autre texte, Griaule précise que l'enfant le conduit sciemment dans la caverne d'un village qui n'est pas le sien (Griaule 1933 : 12). Sa démarche n'est donc pas si ingénue ou naïve.

21. «[Les] gens, s'ils lâchent quelques petits secrets, cachent soigneusement le principal » (Leiris 1981 : 103).

22. C'est d'ailleurs en s'en remettant aux souvenirs du jeune peintre Agagnahou Engueda que Griaule a commencé son enquête sur les jeux éthiopiens, à Paris, en 1926. En pays dogon, en revanche, ses principaux informateurs sur les jeux étaient tous des enfants, du moins avant la guerre. digression tactique. L'enquête, de ce point de vue, est un combat, un siège, une enquête judiciaire ou un interrogatoire policier, avec en face un adversaire à vaincre ou à débusquer, un coupable à confondre ou un suspect à faire avouer, selon des métaphores couramment employées par Griaule ${ }^{19}$. Dans cette lutte pour la vérité, le travail en équipe et le recoupement des « dépositions » sont des stratégies délibérées pour faire parler les uns en les confrontant aux «aveux" des autres (Griaule 1933 : 11-12).

Afin de percer les secrets des adultes, Griaule préconise aussi le recours aux enfants dans l'espoir d'obtenir de leur part de fructueuses indiscrétions, en raison précisément de leur candeur et de leur spontanéité présumées :

L'incompétent qui n'est que très imparfaitement informé donnera parfois des rapports précieux sans se rendre compte de leur gravité. Ainsi un enfant commettra des indiscrétions révélatrices qui se placent parfois à l'origine de la découverte (Griaule 1957 : 56).

Dans ses publications méthodologiques, Griaule donne d'ailleurs quelques exemples de ces indiscrétions enfantines débouchant sur des secrets fabuleux, soigneusement dissimulés par les informateurs adultes. En 1931, alors qu'un "vieil initié [dogon], par respect ou crainte de son Dieu, avait volontairement omis de donner la clé de voûte de l'institution [des masques] ", un " enfant de dix ans la fait découvrir » en conduisant Griaule dans une caverne où sont conservés quatre masques de sept mètres de long (Griaule 1934C : 415). Ce jeune écolier, Abara, est le principal informateur dogon de Griaule sur la thématique du jeu, et son indiscrétion est présentée comme la conséquence de son ignorance et de son " inexpérience ». Extérieur aux mystères des adultes, il " ne pouvait encore saisir toute l'importance des grands secrets dont il secouait quelque peu le voile ${ }^{20}$ " (Griaule 1935 : 5). Partageant l'idée selon laquelle les vieillards seraient les gardiens de secrets impénétrables ${ }^{21}$, Leiris, dans L'Afrique fantôme, mentionne lui aussi quelques découvertes "sensationnelles" à porter au crédit des enfants (Leiris 1981 : 96-97, 163, 172). Dans les années 1930, les enfants innocents, qui font jaillir la vérité, et les adultes coupables, qui la dissimulent, se situent ainsi aux deux pôles de l'enquête ethnographique. Les premiers sont les complices des ethnologues et les seconds leurs adversaires.

Lors des deux premières missions Griaule, l'Éthiopie échappe toutefois à cette vision binaire. Selon Griaule, en effet, ce pays de lettrés chrétiens possède moins des secrets initiatiques à pénétrer que des peintures et des manuscrits à emporter, après les avoir achetés, "troqués " ou commandés auprès de prêtres, de peintres ou d'érudits locaux. Dans ce contexte, les ethnographes ne sont proches ou complices que de leurs hôtes ou collaborateurs adultes, même lors des enquêtes sur les activités ludiques des enfants. Les informations sur les jeux éthiopiens proviennent en effet de jeunes gens cultivés ou de grands adolescents $^{22}$, comme le montrent les fiches et les photographies de Griaule (1935: pl. XIII à XVI, XX à XXV).

\section{AMUSEMENTS RÉCIPROQUES ENTRE GRIAULE ET LES ENFANTS}

Dans les années 1930, les présupposés des ethnographes - sur la candeur ou la primauté des enfants - ne peuvent toutefois suffire à expliquer le poids initial des jeux dans les travaux personnels de Griaule. Si Leiris ou Mouchet ont souvent interrogé de jeunes écoliers, et si Eric Lutten s'est intéressé ponctuellement 


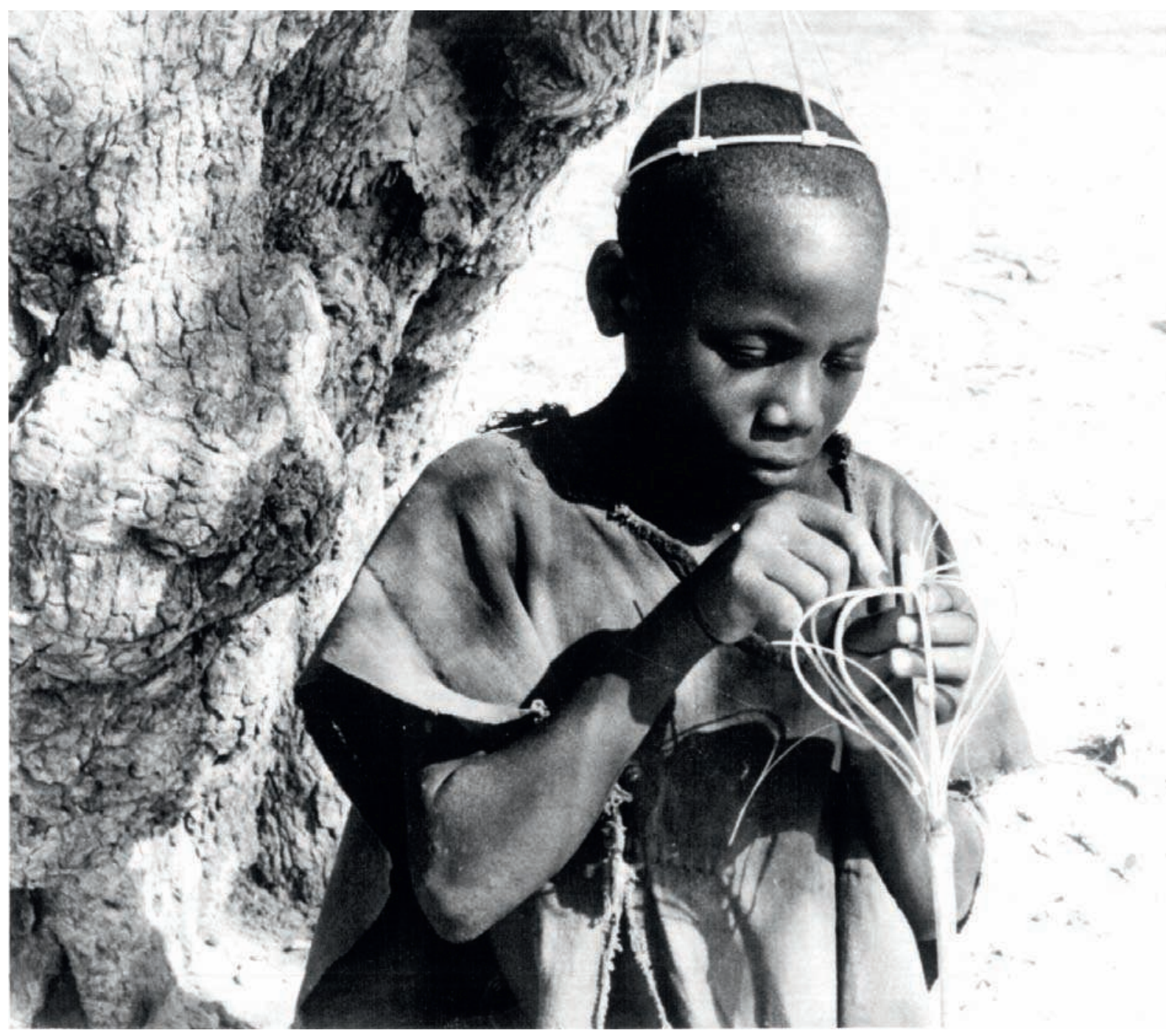

Fig 5 Koguèm armant son fusil en tige de mil (mission SaharaSoudan, 1935. Fonds MarcelGriaule, bibliothèque Éric-deDampierre, MAE, Université de Paris Ouest, Nanterre, La Défense).

-

23. Lutten 1933 et 1934.

24. Chastenet $1957: 21$.

25. Voir notamment : Leiris 1981 : 173; Griaule 1957b : 154.

26. Note de Leiris dans l'agenda de Dakar-Djibouti, 29 septembre 1931 (fonds Marcel-Griaule). aux poupées sous un angle plutôt muséographique ${ }^{23}$, Griaule est en effet le seul à avoir réservé aux jeux une part importante de ses recherches et de ses publications. Deux raisons subjectives, liées à sa personnalité, l'ont sans doute incité à choisir ce thème : un regard paternel et amusé sur le monde des enfants, et un penchant pour leur imaginaire merveilleux.

Personnage charismatique, Griaule est aussi un «incomparable amuseur d'enfants ${ }^{24}$ », en France comme en Afrique. Aux détours d'un écrit ${ }^{25}$ ou d'une photographie, on le découvre traînant derrière lui une ribambelle de gamins ou saisissant la moindre occasion pour chatouiller la plante des pieds d'un jeune enfant, asseoir sur ses genoux une fillette médusée ou jouer avec le fils de l'esclave éthiopienne Dasta (petit garçon turbulent qui, par dépit, lui jettera une pierre à l'annonce de son départ). Quant à l'affection paternelle de Griaule pour ses jeunes informateurs, elle ne fait aucun doute : elle s'exerce notamment vis-àvis des deux élèves dogon - Abara (douze ans) et Amadigné (treize ans) - envoyés par l'instituteur dès les premiers jours d'enquête à Sangha, en $1931^{26}$. Pour ses 


\section{-}

27. Qu'il s'agisse d'adultes ou d'enfants, les informateurs et interprètes des missions Griaule sont tous rétribués. Les noms de quatre enfants dogon (Abara, Koguèm, Alabouri et Kéné] apparaissent d'ailleurs sur une fiche de paye mensuelle datée du 28 février 1935 (fichier dogon 1931-1937 du fonds MarcelGriaule). Salariés de la mission au même titre que leurs aînés, les petits informateurs de 1931 sont tous assis au premier plan de la photographie regroupant l'ensemble des ethnographes et de leurs collaborateurs dogon (voir fig. 4).

28. Leiris $1981: 112,123$.

29. Au Cameroun, Lebeuf avoue avoir transformé une collecte de poupées en «jeu de cache-cache » avec des fillettes, et une collecte d'insectes en amusement gratuit avec de jeunes garçons (Lebeuf 1945 : 40, 168).

30. Fonds Marcel-Griaule. recherches sur les jeux dogon, Griaule travaille directement avec eux, ou avec le petit Iréko, en les rémunérant ${ }^{27}$, mais aussi en veillant sur eux et en acceptant de leur part une proximité qu'il refuse aux adultes. En 1931, Abara est d'ailleurs un habitué du campement de la mission. Rejoint parfois par Iréko, Amadigné et Binèm, il y mange et $\mathrm{y}$ dor ${ }^{28}$, protégé par Griaule :

Ce matin, Griaule a chassé à coups de cravache un marchand bambara qui avait voulu prendre la nourriture d'un de nos petits informateurs (qui mangeait chez nous) (Leiris $1981: 109$ ).

Abara est de toutes les «tournées ethnographiques » de Griaule autour de Sangha, d'abord avec Amadigné en 1931, puis avec Koguèm (alors âgé de douze ans) en 1935. Mais l'attachement réciproque entre Griaule et le benjamin de ses informateurs se manifeste avec davantage d'acuité au moment de leur séparation (en 1931) puis de leurs retrouvailles (en 1935). Le jour du départ de la mission Dakar-Djibouti, Abara se poste discrètement au bord de la route pour demander à Griaule de l'emmener avec lui, avant de faire demi-tour en pleurant, malgré la montre offerte en cadeau d'adieu (ibid. : 129). Trois ans plus tard, lorsque Griaule revient à Sangha, il commence fébrilement par chercher des yeux le jeune Abara, qui n'a guère grandi : «bouleversé, le cherchant parmi les têtes qui lui arrivent au menton, Griaule le découvre soudain à la hauteur de son coude» (Gordon 1935, I : 2). La seconde séparation, en 1935, est en revanche moins pénible, voire franchement comique. La nuit du départ, les petits informateurs embauchés cette année-là paradent dans le campement en arborant les habits abandonnés à leur intention par les ethnologues des deux sexes : Abara dans un pyjama molletonné offert par Griaule, Alabouri avec un chausson rouge d'Hélène Gordon, Koguèm dans un boléro bleu et Kéné en bas de laine (ibid., VIII : 2).

Cette scène cocasse peut paraître anecdotique, mais elle témoigne d'une qualité appréciée et recherchée par Griaule : la capacité des enfants à introduire de la fantaisie dans la vie austère et bien réglée des ethnographes, ou même leur aptitude à faire glisser l'enquête vers le jeu, sans rien perdre de leur efficacité. Alors qu'il ne plaisante guère avec le travail des adultes, Griaule est bien souvent le complice d'un tel glissement (à l'instar de son collègue Lebeuf au Cameroun et au Tchad). Avec les enfants, Griaule et Lebeuf conjuguent sans cesse amusement et travail, quelle que soit la nature de ce travail ${ }^{29}$. Bien sûr, pour les reconstitutions de jeux à des fins d'observation et de photographies, cette combinaison va déjà de soi, comme le prouvent les mines réjouies d'Abara et de ses camarades sur les nombreux clichés pris par Griaule en 1931. Dans le prolongement de ces reconstitutions ludiques, Griaule imagine une séance hilarante de photographies publicitaires à destination d'un sponsor de la mission Dakar-Djibouti. Sollicitant à nouveau les talents d'acteur des jeunes Abara, Amadigné et Iréko, il les photographie à deux reprises se ruant joyeusement sur des boîtes de conserve Amieux déposées au premier plan (voir fig. 2).

Les jeux et les mots d'enfants enchantent Griaule, dans son travail comme dans le cadre de ses voyages ou de sa vie familiale, et il en saisit certains dans son calepin personnel, en les ajoutant aux citations, aphorismes et métaphores qu'il collectionne. Sur des pages de carnets correspondant aux années 1935$1937^{30}$, il note aussi bien les réflexions candides de ses filles que les amusements enfantins dont il est le témoin lors d'un passage à Alger : 


\section{-}

31. Voir, par exemple, la ronde des cigognes ou le lancer du javelot (Griaule 1934b : 63, 163-164; 1935 : 71, 92-94).

32. «M. Marcel Griaule nous montre [...] un garçonnet charger sur les épaules un jeune crocodile qu'il rapporte à ses parents. Ses traits ont l'amabilité, la familiarité d'un petit gars des provinces françaises, joyeux d'avoir pêché une belle carpe » (Barrucand 1935).

33. Radio-conférence du 25 juin 1935 (texte dactylographié, archives du musée de l'Homme, C2AM1C8a, n 10).

\section{Décrite également dans} l'ouvrage sur les jeux éthiopiens (Griaule 1935 : 17-18), cette invention renvoie aux propres rêves d'aviateur de Griaule, et son évocation laisse poindre une vision romantique et nostalgique de l'enfance.

35. Griaule $1938: 1,3,28$.

Fig. 6 Le départ de Mamadou Keita avec la mission DakarDjibouti (Bamako, 1931, photographie de Marcel Griaule. Musée du quai Branly).
Tiany [Christiane] : Celui-là a zéro bras. Je n'en veux pas (une poupée cassée). [...]

Un gosse qui joue au traîneau assis sur un plat d'émail.

Enfants faisant [des] graffiti avec [leur] crachat sur [une] porte et raclant avec une baguette (rue François-Villon).

Ces quelques phrases témoignent assez bien de la fascination qu'éprouve Griaule pour un imaginaire enfantin capable de construire un monde merveilleux à partir de rien, ou presque rien, en transformant, le temps du jeu, un crachat en dessin, un plat d'émail en traîneau, une poupée loqueteuse en adorable bébé (du moins tant qu'il lui reste un bras). Mais ce monde «magique » de l'enfance, qui l'amuse ou l'émerveille, il lui est difficile de le prendre au sérieux en raison même de sa fantaisie. En revanche, il en "joue" afin de rendre plus vivants ses récits littéraires et ses conférences. Dans Les Flambeurs d'hommes, Griaule utilise ainsi ses fiches ou son manuscrit sur les jeux éthiopiens pour introduire des épisodes ludiques entre deux événements dramatiques ${ }^{31}$. Il se sert également des photographies d'enfants dans ses conférences sur les Dogon, à la fois pour égayer son exposé et donner une image plus familière et sympathique des Africains ${ }^{32}$. En 1935, enfin, il fait appel à ses trois filles (alors âgées de sept, neuf et onze ans) pour animer une conférence radiophonique sur les « jeux abys$\operatorname{sins}^{33}$ » et lui insuffler une fraîcheur tout enfantine. Dialoguant et plaisantant affectueusement avec elles, il leur pose des devinettes et les encourage à parler de leurs propres jeux, avant de les comparer à ceux des petits Éthiopiens. Il célèbre par ailleurs l'imagination fertile des enfants, indissociable de leur univers onirique, en évoquant longuement la « machine à voler » inventée par l'un de ses informateurs éthiopiens lorsqu'il était enfant ${ }^{34}$. Inaugurée par un dialogue ludique sur les jeux, cette conférence radiophonique se transforme toutefois, dans sa seconde moitié, en un récit flamboyant de l'expédition de Griaule à dos de mulets. Comme dans Les Flambeurs d'hommes, les amusements insouciants des enfants introduisent donc aux drames, aux mésaventures ou plus généralement au monde "sérieux" des adultes. Activité "délectable" mais "mineure35", les jeux sont, pour Griaule, une porte d'entrée privilégiée ou un prélude nécessaire, dans ses récits comme dans ses recherches.

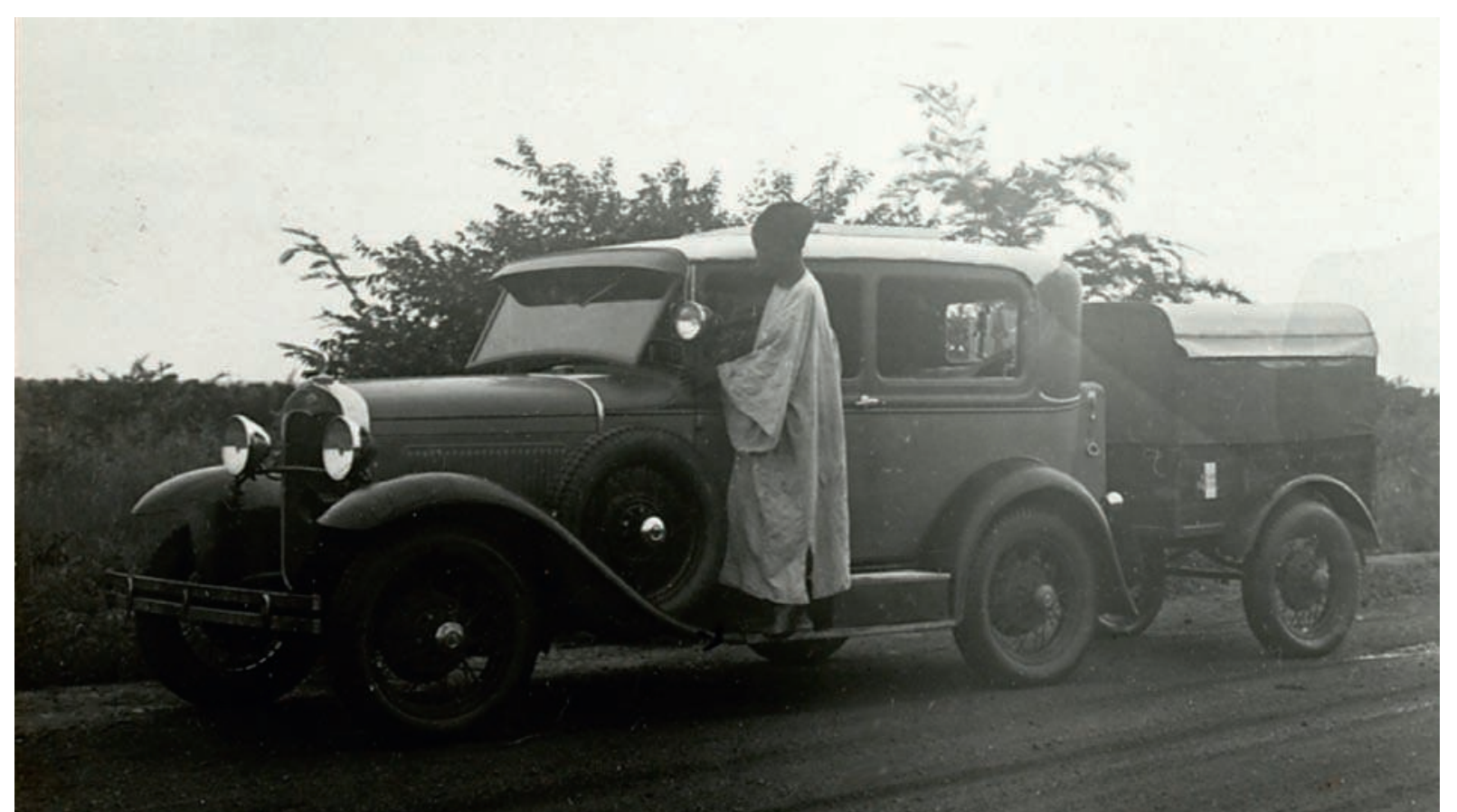


Le premier ouvrage ethnographique de Griaule traite des dessins d'enfants, systématiquement rangés parmi les jeux dans ses fichiers comme dans ses monographies. Publié en 1933, le livre porte plus précisément sur les Silhouettes et graffiti abyssins recueillis par Griaule sur les murs des églises du Gojjam, lors de sa première mission. De «jeunes garçons» en seraient les auteurs, selon lui. Or, à travers une telle attribution, hypothétique et probablement erronée, Griaule dévoile une conception des productions enfantines directement héritée de Mauss. Dans l'introduction de l'ouvrage, cosignée Mauss et Griaule, les deux chercheurs évoquent sans ambiguïté un "style [de dessins] qui est le produit d'une activité et d'un art strictement enfantins ${ }^{36}$ ", opposés à ceux des adultes. Mais ils déduisent cette affirmation de leur interprétation stylistique et non des observations de Griaule. De leur point de vue, la «licence " artistique ou la liberté expressive qui transparaît dans ces dessins ne peut être associée qu'aux enfants et à leur "espièglerie", par opposition au classicisme et à la fixité de «l'art adulte» :

Le jeune abyssin est libre à l'intérieur de son expression. Au contraire, dans les mêmes églises où l'espièglerie de la jeunesse se donne large champ, l'art adulte est figé dans un style proprement abyssin, certes, mais dogmatique, et, au fond byzantin. Cette opposition entre l'art des deux âges est un phénomène profond (Mauss et Griaule 1933: 6).

Mauss et Griaule reconnaissent tout au plus que les "silhouettes " peintes correspondent à un style intermédiaire entre l'art enfantin des graffiti, qui échappe à toute règle, et les peintures religieuses des adultes, prisonnières de conventions immuables. C'est pourquoi ils les attribuent à de jeunes apprentis (ibid.), en suggérant que l'art des enfants évolue avec l'âge d'une expérimentation autonome vers un premier apprentissage normatif. Dans le chapitre qui suit l'introduction, Griaule semble toutefois moins catégorique : il affirme que ces silhouettes aux allures d'ombres chinoises, incorporées dans un damier blanc et noir, "sont généralement l'œuvre d'apprentis peintres ", tout en concédant que certaines d'entre elles sont exécutées par des artistes renommés (Griaule 1933b : 9). Les faits obligent pourtant à inverser cette proposition : toutes les silhouettes reproduites dans le livre ont en effet pour auteur un peintre connu et âgé qui décorait l'église de Zärä Buruk ${ }^{37}$, alors que Griaule n'apporte en revanche aucun témoignage visuel ou oral permettant d'attribuer ce type de peinture à un jeune apprenti. Mais, à ses yeux, ce style libre et simple, proche de l'esquisse ou de l'ébauche, porte forcément la signature de l'enfance ou du moins de l'adolescence, d'autant que les motifs représentés sont de taille réduite et s'inspirent de la vie quotidienne ou de la nature, et non de la Bible. En apparence terre à terre, cette peinture est donc interprétée comme une activité mineure ou un « délassement sans importance " réservé aux jeunes élèves (Griaule 1930b : 369).

Grâce aux recherches de Claire Bosc-Tiessé (2002), on sait aujourd'hui que ces peintures en damier au style si particulier imitent les carreaux de faïence hollandais qui ornaient l'église royale de Owesqwam avant sa destruction. Représentant des paysages en bleu sur fond blanc, ces carreaux ont tellement marqué les esprits qu'ils ont servi de modèle aux «silhouettes » encadrant les portes ou les fenêtres des églises éthiopiennes ${ }^{38}$. Griaule avait donc raison de voir 
- •

42. La figure 29 publiée par Griaule (1933b : 25) est une copie précise de la figuration classique d'un «prêtre du ciel » (communication écrite d'Anaïs Wion). Quel que soit l'âge du dessinateur, il est donc difficile d'opposer ce type de graffiti à la peinture religieuse éthiopienne.

43. Sur une même fiche de Griaule, le dessin 80 de son ouvrage de 1933 (ibid. : 33) figure à côté d'une phrase en amharique ayant valeur de signature et d'ex-voto : «L'honorable serviteur du Ras Bayana Tembay [?] a fait cette porte, qu'une récompense lui soit donnée » (entrée «dessin »du second fichier éthiopien, fonds Marcel-Griaule).

44. Griaule $1938: 1$

45. C'est en septembre 1929, au retour de cette première mission, que Griaule obtient son diplôme à I'Institut d'ethnologie.
Or, en dépit de leur modestie, ces dessins religieux, tracés au charbon de bois, ont été exécutés par des adultes. Quant aux graffiti anonymes ornant les murs extérieurs, accessibles à tous, ils peuvent être l'œuvre de jeunes écoliers délurés, mais aussi de fidèles plus âgés reproduisant maladroitement des peintures religieuses ${ }^{42}$. Du reste, de tels dessins voisinent parfois avec des prières en amharique griffonnées par les bâtisseurs ou les rénovateurs de l'église ${ }^{43}$.

Les analyses et les hypothèses développées par Griaule à propos des silhouettes et des graffiti éthiopiens révèlent ainsi, en creux, sa vision des productions artistiques des adultes, par opposition à celles des enfants. Dans le contexte de sociétés holistes, ou perçues comme telles, Griaule dénie aux adultes, et $a$ fortiori aux peintres confirmés, toute liberté créative. Leurs peintures ou leurs dessins ne peuvent être que des œuvres travaillées, soignées, complexes, sérieuses, conventionnelles et chargées de sens (biblique, mythologique ou ésotérique). Dès lors que ces qualités font défaut, il ne peut s'agir, en revanche, que de jeux ou de brouillons d'enfants, synonymes de divertissements gratuits ou d'apprentissage.

\section{UNE VOIE D'ACCÈS VERS LE MONDE FERMÉ DES ADULTES}

Des analyses précédentes, on peut tirer plusieurs conclusions quant à la place que Griaule réserve à la culture enfantine dans l'ensemble de son œuvre. Avant la guerre, Griaule considère les jeux comme des « amusements mineurs44 », indépendants des adultes et de leurs règles (ou opposés à eux). Sont incluses dans cette catégorie toutes les activités et productions propres au monde initial de l'enfance, défini comme un univers "merveilleux» et "protégé " (Griaule 1938: 27-28). Si ces jeux sont antérieurs et inférieurs aux activités sérieuses et religieuses des adultes, ils y préparent toutefois, dans la mesure où ils représentent une phase d'expérimentation ou d'apprentissage plus ou moins autonome, extérieure à l'école et à "son système de dressage " (ibid.).

Or, dans son propre parcours de recherche, Griaule semble suivre ce même apprentissage conduisant par étapes ascendantes du socle des jeux enfantins jusqu'à la clé de voûte des connaissances religieuses, en allant toujours du plus simple au plus complexe, des interlocuteurs les plus jeunes aux plus âgés, de l'information la plus accessible aux secrets les mieux gardés. Quand il commence à travailler sur les jeux éthiopiens, en France puis lors de la mission de 19281929, Griaule n'est encore qu'un étudiant s'exerçant aux techniques d'enquête ${ }^{45}$. Deux ans plus tard, lorsqu'il entreprend ses recherches sur les jeux dogon dans le cadre de l'expédition Dakar-Djibouti, il prolonge cette expérimentation en éprouvant sur le terrain les méthodes apprises à l'Institut d'ethnologie. Enfin, une fois nommé professeur d'ethnologie en 1942, il se tourne définitivement vers l'étude des connaissances supérieures des vieillards dogon, qualifiés de "docteurs " (Griaule 1952C : 34). Le sommet est donc atteint, sur tous les plans et simultanément.

Une telle ascension se dessine très tôt : dès 1931, Griaule place clairement les jeux au pôle inférieur de ses recherches et les phénomènes religieux au pôle supérieur. Une phrase prononcée avant Dakar-Djibouti trahit d'ailleurs cette bipolarité en voulant souligner l'exhaustivité des enquêtes prévues: "Nous tenterons de suivre les improvisations grandioses des prêtres parlant à leurs dieux, comme celles des enfants au cours de leurs jeux" (Griaule 1931). En 1938, cette hiérarchisation thématique est confirmée lors du doctorat de 
Fig. 8 Hélène Gordon et Solange de Ganay entourées d'enfants dogon (mission Sahara-Soudan, 1935. Fonds Marcel-Griaule, bibliothèque Éric-de-Dampierre, MAE, Université de Paris Ouest, Nanterre, La Défense).
Griaule, avec une thèse principale sur les Masques dogons et une autre, secondaire, sur les Jeux dogons. Mais la minoration des jeux au profit du «sacré » était déjà perceptible dans des publications antérieures. Dans le numéro de Minotaure consacré à la mission Dakar-Djibouti, Griaule signait un article sur les funérailles dogon (1933c) et un autre sur les peintures religieuses éthiopiennes (1933d), tandis que les jeux n'étaient représentés que par une unique photographie de poupée.

D'un point de vue méthodologique, Griaule attend toutefois le début des années 1950 pour justifier et théoriser a posteriori la progression de ses enquêtes depuis les enfants dogon jusqu'aux vieillards. Il y est poussé par le besoin de démontrer l'existence de couches successives du savoir, seules capables d'expliquer les variantes mythologiques recueillies, du moins selon l'hypothèse retenue d'une cosmogonie dogon uniforme et figée. Dans un article sur «l'enquête orale en ethnologie » publié en 1952, Griaule préconise ainsi de mener des «investigations successives dans des couches de plus en plus instruites de la population ", en progressant par rang d'âge ou d'initiés, et en partant toujours du bas, c'est-àdire des enfants :

En cette matière, comme en matière d'enseignement chez les Occidentaux, il convient de suivre une progression régulière allant de l'élémentaire au complexe, quitte à ne découvrir les principes directeurs qu'en fin d'instruction. Pratiquement, il ne faut utiliser les informateurs "savants " qu'après avoir épuisé un grand nombre d'éléments de moindre importance (Griaule 1952 : 545-546).

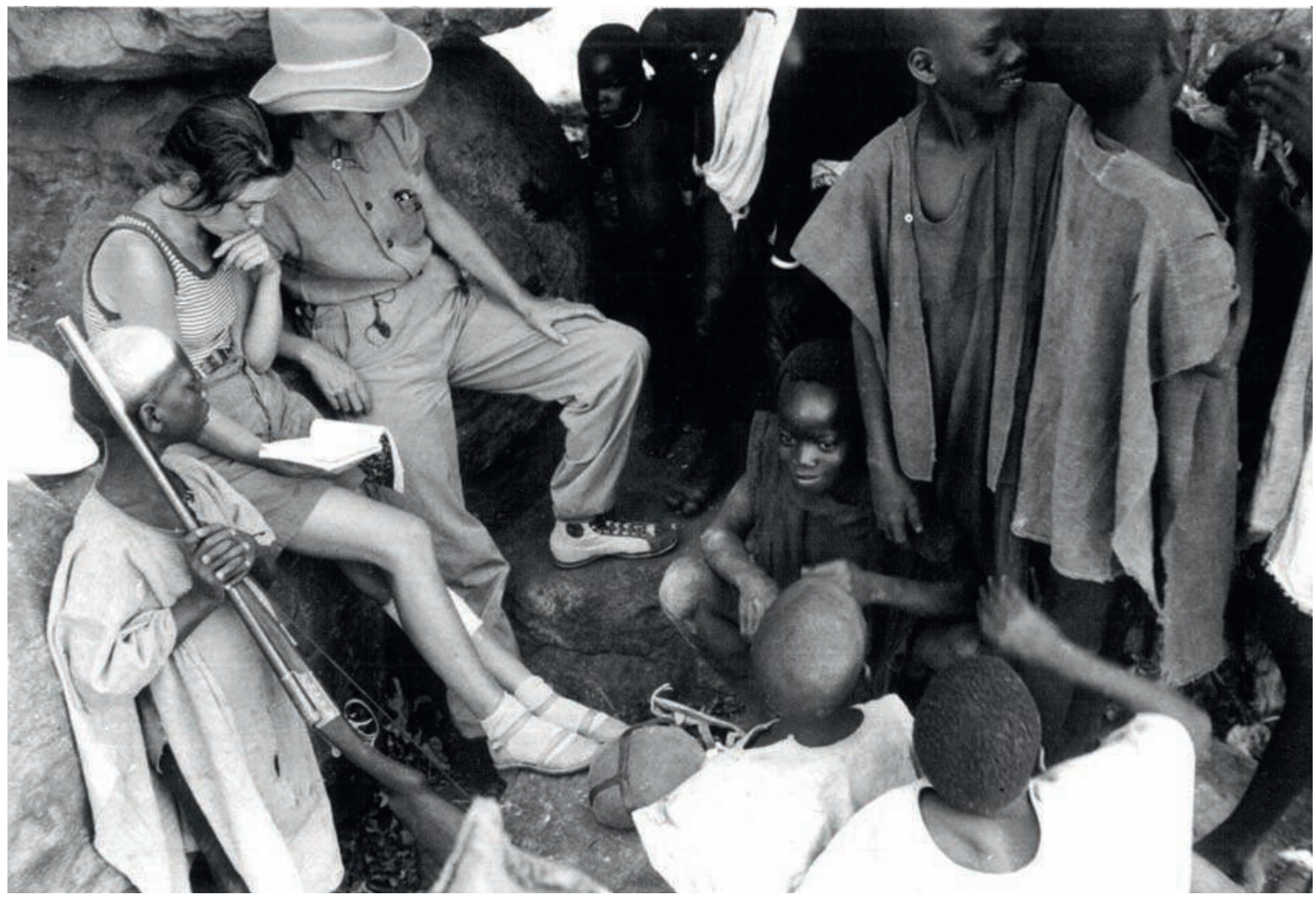


Fig. 9 Cheval en tige de mil. Fiche de Griaule classée à l'entrée «jeu» du fichier dogon (fonds Dakar-Djibouti, bibliothèque Éric-de-Dampierre, MAE, Université de Paris Ouest, Nanterre, La Défense).

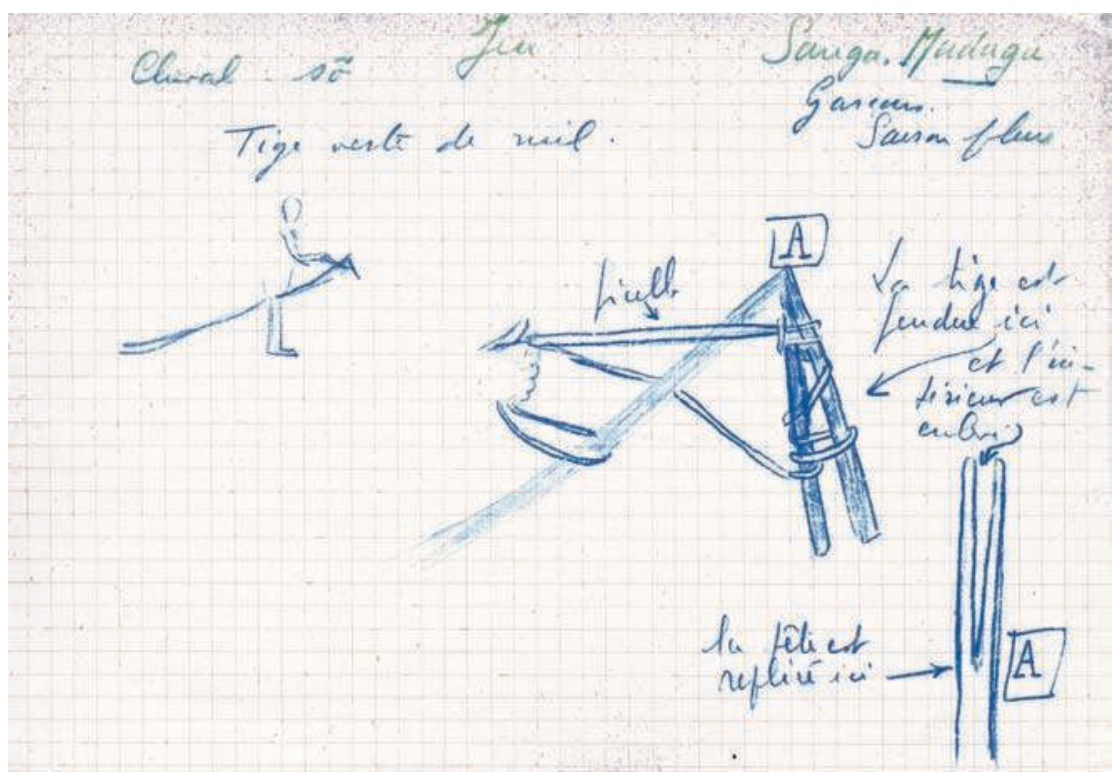

Griaule continue donc à faire des enfants le point de départ de toute enquête, mais dans une simple perspective pratique et stratégique: il les considère comme l'unique porte d'entrée permettant d'accéder sans effraction au monde fermé des adultes et à leurs secrets. À partir des années 1940, en revanche, il renonce à étudier les jeux pour eux-mêmes et cesse simultanément de les présenter comme des activités opposées à celles des adultes.

\section{Le dénouement initiatique : l'accès au savoir supérieur des vieillards (1946-1956)}

Après la guerre, dans le milieu des ethnologues africanistes, l'heure n'est plus à l'inventaire rapide et exhaustif des sociétés colonisées. À un archivage urgent, dans un esprit de sauvetage, succède une volonté de valorisation des cultures et des "systèmes de pensée " africains. Griaule s'inscrit dans cette évolution et se fixe un nouvel objectif : gravir les plus hautes marches du savoir ésotérique local afin d'atteindre le système mythologique ou symbolique qui coiffe, selon lui, chaque société africaine ${ }^{46}$. Griaule parle également de pénétrer les couches les plus profondes de ce savoir pour en atteindre le cœur, selon une seconde métaphore évoquant l'image d'une sphère religieuse refermée sur ses secrets. Dans tous les cas, cette progression vers le haut ou vers le centre oblige Griaule à s'éloigner des enfants pour se rapprocher des vieillards ou des "grands initiés " dogon. Les premiers, détenteurs de connaissances rudimentaires et fragmentaires, sont situés à la base de la pyramide et aux marges de la sphère religieuse, tandis que les seconds, au sommet, sont perçus comme les dépositaires d'un savoir synthétique et complet qui, sous une forme cosmogonique, régirait l'ensemble de la société47. En glissant ainsi de l'imaginaire enfantin aux mythes des «savants», Griaule n'en poursuit pas moins sa quête du merveilleux, avec sérieux cette fois et non plus avec tendresse ou amusement. En outre, il n'abandonne ni ses ambitions littéraires, ni son goût pour les dessins, ni même son intérêt pour les jeux d'enfants, mais il les utilise désormais comme éléments d'illustration ou de narration du récit mythologique qu'il construit graduellement. 
En 1946, la rencontre avec le vieil aveugle Ogotemmêli s'avère déterminante. Ce «savant " présumé comble les attentes de Griaule en se chargeant tout à la fois de «révéler " une cosmogonie et d'en faire l'exégèse. Entre le 20 octobre et le 2 décembre, Griaule a des entretiens quotidiens ou biquotidiens avec cet informateur dogon plein d'assurance qui lui a librement offert ses services, par des moyens détournés ${ }^{48}$. Les relations entre enquêtés et enquêteurs s'en trouvent modifiées, mais davantage dans l'esprit que dans la forme. Vis-à-vis des ethnographes, Ogotemmêli n'est plus ni un suspect qu'il faut faire parler, ni un adversaire contre lequel il faut lutter afin de briser ses défenses. Griaule le présente plutôt comme un "initié de haut rang " instruisant progressivement un élève, ou encore comme un "partenaire", engagé, avec l'enquêteur et son interprète, dans un travail de synthèse et d'explication 49 .

Après la mort d'Ogotemmêli, en 1947, Griaule poursuit son «initiation » auprès d'une poignée d'informateurs âgés ou "instruits", en particulier Ongnonlou, Manda et Yébéné. Or la plupart de ces initiateurs sont des habitués des enquêtes menées par Griaule et son équipe : le "prêtre " Yébéné est leur informateur depuis 1937, tandis que son collègue Manda a été approché dès 1935. Par ailleurs, Amadigné et Koguèm (deux des petits informateurs embauchés au début des années 1930 à l'âge de de douze ou treize ans) deviennent après guerre de proches collaborateurs de Griaule, cumulant les fonctions d'interprètes, d'enquêteurs et d'informateurs, tant en pays dogon que dans les régions voisines. Comme le montrent ces exemples individuels, la durée des recherches menées en pays dogon, de 1931 à 1956, a une incidence sur le changement de statut des informateurs. De plus en plus familiers des ethnologues (et de leurs travaux), les informateurs des années 1930, petits et grands, forment quinze ou vingt ans plus tard un noyau d'informateurs expérimentés, rompus à l'enquête et dévoués aux enquêteurs. Leur avancement en âge leur a permis d'accroître leurs connaissances, en partie au contact de Griaule, et les ethnographes peuvent donc progresser vers les couches supérieures du savoir sans avoir à changer d'interlocuteurs. Mais les informateurs ne sont pas les seuls à vieillir. En 1946, Griaule n'est plus le jeune père qui s'amusait aussi bien avec ses enfants qu'avec les petits Koguèm ou Amadigné. L'une de ses filles ${ }^{50}$, âgée de vingt-deux ans, l'accompagne d'ailleurs lors de cette mission, avec un statut de collaboratrice chargée de la langue dogon, tandis que Koguèm, qui a pratiquement le même âge, sert d'interprète lors des entretiens avec Ogotemmêli.

En tout état de cause, les amusements ne sont plus de mise au moment où Griaule élabore, à la faveur de synthèses successives, un système cosmologique cohérent et uniforme. Depuis qu'il est devenu «élève», Griaule dépend entièrement de ses informateurs privilégiés, lesquels en profitent pour prendre des libertés par rapport au cadre initial de l'enquête. Devant une telle attitude, l'irritation de Griaule se traduit par une série de commentaires ou de formules ironiques notés au crayon dans ses carnets de terrain. L'une des expressions qui reviennent le plus souvent témoigne bien de sa contrariété face à l'importance grandissante que prennent (ou se donnent) ses informateurs privilégiés. Sur le registre de $1953^{51}$, Griaule note à plusieurs reprises, pour expliquer le report ou la lenteur d'une enquête: "Ces Messieurs sont fatigués par la bière d'hier »; "ces MM parleront cet après-midi "... Aux enfants espiègles et rieurs des années 1930 ont succédé des messieurs savants, mais agaçants. Quant aux jeux, désormais

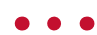

48. Jolly 2001-2002 : 93-94.

49. Griaule 1952 : 545-546.

50. II s'agit, bien sûr, de Geneviève Calame-Griaule, future ethnolinguiste.

51. Cf. p. 646, 678, 596 (fonds Marcel-Griaule). 
- $\bullet$

52. L'une de ces fiches a été rédigée le 28 septembre, l'autre le 10 novembre 1946 (fonds MarcelGriaule].

53. « L'enfant ne peut rien faire de sérieux 》; «rien en eux n'est fixé »; «ils ont leur vie à eux » et «ne rêvent que désordre » (Griaule 1948 : 184-188).
Fig. 10 Jeu de ficelle. Fiche de Griaule classée à l'entrée "jeu " du fichier dogon (fonds DakarDjibouti, bibliothèque Éric-deDampierre, MAE, Université de Paris Ouest, Nanterre, La Défense). relégués à l'arrière-plan de cosmogonies immuables, ils se trouvent insérés à des fins de narration ou de classement dans une trame de nature mythologique ou symbolique.

\section{DES JEUX TERRE À TERRE AUX MYTHES COSMOGONIQUES}

En 1946, s'il ne mène plus d'enquête orale sur les jeux, Griaule continue à noter certains amusements enfantins observés à l'improviste. Le fichier dogon constitué cette année-là comporte ainsi une entrée " jeu » qui comprend six fiches, dont quatre de Griaule. Deux d'entre elles seront utilisées dans Dieu d'eau (1948) pour donner davantage de couleur et de cohérence au récit mythologique dévoilé jour après jour par Ogotemmêli. Au début du chapitre correspondant à la vingt-quatrième journée d'entretiens, Griaule s'inspire en effet de ces deux fiches ${ }^{52}$ pour dépeindre l'univers ludique des garçons incirconcis, avant d'aborder les événements mythiques ayant abouti à la circoncision. Il décrit notamment des «bambins nus " s'amusant à frapper les chiens avec de longues tiges de mil, ou des enfants jouant avec leur troupeau de sauterelles aux ailes brisées (Griaule 1948: 184). L'image qui se dégage de ce tableau, ou des citations qui le complètent ${ }^{53}$, est conforme à la conception que Griaule et Ogotemmêli ont de l'enfance : un espace autonome de liberté, de divertissement et d'espièglerie situé en marge du monde ordonné, sérieux et figé des adultes. Après Les Flambeurs d'hommes en 1934, les notes ethnographiques sur les jeux sont donc de nouveau utilisées à des fins narratives, cette fois pour introduire un récit mythologique.

$\mathrm{Au}$ cours de la mission suivante, en 1948, Griaule reprend son enquête sur les jeux dogon afin de les réexaminer à l'aune du mythe. Partant de son propre inventaire publié en 1938, il interroge Koguèm et Ankundyo sur le sens symbolique ou cosmologique de chaque jeu. Cette année-là, l'objectif n'est pas encore d'obtenir de longs développements explicatifs. Avec ses interlocuteurs dogon, tous adultes, Griaule cherche à associer un jeu à chaque épisode du mythe recueilli deux ans auparavant. Cela l'oblige, dans un second temps, à effectuer un classement

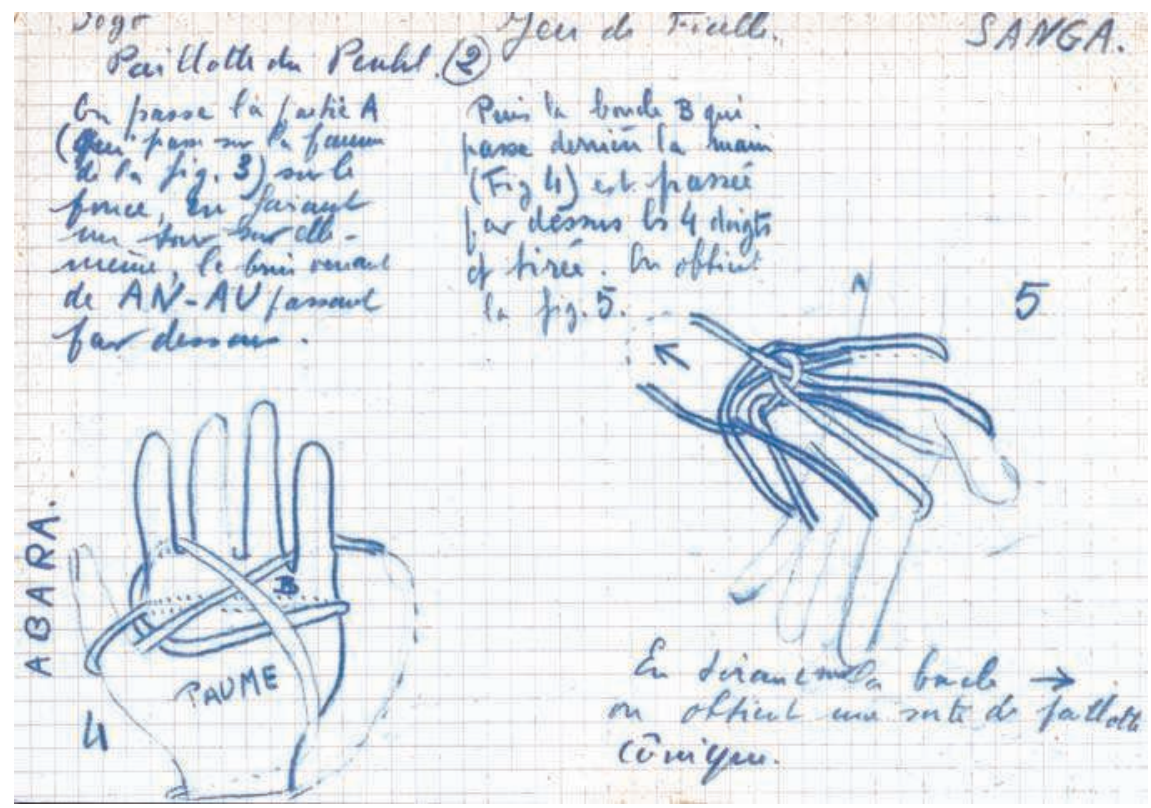


pour que «tous ces jeux reproduisent dans l'ordre les aventures du [Dieu d'eau] $\mathrm{No}[\mathrm{mmo}]^{54}$ » telles qu'elles ont été relatées par Ogotemmêli. Même lorsqu'elles sont d'inspiration étrangère, les productions enfantines reçoivent elles aussi une signification en relation avec cette histoire mythique : "chapeau canotier : les mains du No[mmo] en abat-jour sont les yeux pour voir le monde à la descente ${ }^{55}$ ". En raison de leurs liens présumés avec l'entité mythique qui a ordonné le monde, les jeux ou les jouets prennent par ailleurs une coloration nouvelle sur le plan social. Considérés jusqu'alors comme des activités sans importance, libres et indépendantes, brouillonnes ou désordonnées, ils participent désormais à l'organisation du monde ou du village : «le jeu organise le village »; « jouer = remettre constamment le monde dans l'organisation de Dieu ${ }^{56}$ ".

$\mathrm{Au}$ cours des missions suivantes, Griaule continue, chaque fois qu'il enregistre une nouvelle version de la cosmogonie dogon, à réactualiser l'interprétation savante des jeux enfantins. En 1953, Ongnonlou, Manda et Ambara introduisent une complexité supplémentaire en répartissant la totalité des jeux dans trois «familles» correspondant aux trois entités marquantes du mythe dogon: Amma, qui a créé le monde, Nommo, qui l'a organisé, et enfin Yurugu, qui l'a perturbé57. De par ce classement, chaque jeu est le reflet présumé d'un épisode mythique impliquant l'une ou l'autre de ces trois entités. C'est ainsi que la ronde ãyo, rangée dans la seconde famille, «imite la rentrée du No[mmo] dans l'eau à la descente de la caisse ${ }^{58}$ ». Cette volonté d'ordonner les jeux s'accompagne de la quête de modèles prototypiques censés reproduire le mythe et obéir à ses canons. Il faut rappeler que, selon le point de vue exprimé par Griaule après la guerre, le système cosmogonique régit jusqu'au jeu des enfants, dont il détermine le déroulement et l'ordonnancement. Dans cette logique, les jeux qui se dissocient du mythe ne peuvent être que des variantes dégradées, privées de sens, ou des versions simplifiées masquant sciemment une vérité ésotérique. S'il reste convaincu d'une origine religieuse des jeux, Griaule renverse donc sa proposition initiale : après avoir pensé que les amusements des enfants gardaient la trace de mythes disparus, il considère désormais que ce sont les mythes qui conservent la mémoire des jeux ou des jouets dans leur version originelle.

L'exemple le plus significatif est le jeu dogon walia, appelé aussi in et connu ailleurs en Afrique sous le nom d'awele. Lorsque l'objet est sculpté, il s'agit d'une augette en bois comportant deux rangées parallèles de six cases creuses dans lesquelles les joueurs déplacent leurs pions. À la suite d'une enquête menée en août 1948 auprès du "prêtre" Manda, celui-ci établit un lien symbolique entre l'arche mythique et ce jeu, qui, précise-t-il, devrait toutefois compter seize trous et non douze pour figurer deux rangées d'ancêtres mythiques, mâles et femelles ${ }^{59}$. Quelques mois plus tard, en décembre, Manda et Ongnonlou adoptent une perspective plus cosmologique en associant les douze cases réglementaires aux douze lunaisons et les pions aux étoiles; ils précisent également que ce "jeu fait du bien au monde ${ }^{60} »$. Dans un article daté de 1948 sur l'arche du monde et ses représentations matérielles, Griaule s'aligne immédiatement sur cette double interprétation. Avec ses douze trous, le jeu actuel « reproduit et facilite la marche de l'univers ", écrit-il (Griaule 1948b : 122), mais il est aussi l'image retouchée et simplifiée de l'arche du monde et de ses seize compartiments :

Cette simplification est destinée avant tout à cacher au profane le sens du symbole en disposant une série de relais entre l'image mythique [...] et l'objet qui le rappelle (ibid. : 123).
- -

54. Note 1 de la fiche 21 du 30 août 1948, entrée «jeux » du fichier de 1948 (fonds Marcel-Griaule). Sur cette même fiche, les jeux sont classés par numéros : «jeu $\mathrm{n}^{\circ} 4$ = parcours du $\mathrm{N}[\mathrm{ommo}]$ dans le ciel pour sortir ; jeu $n^{\circ} 5=[\ldots]$ sortie du Nommo; jeu $n^{\circ} 6$ (lutte ajori) = Réveil du Lebe ».

55. Fiche 23 du $1^{\text {er }}$ septembre 1948 (ibid.).

56. Fiche 26 du 23 septembre 1948 (ibid.).

57. Cette classification est annoncée au conditionnel dès 1949 ( fiche «Création A88 » du 5 janvier 1949, classée dans le paquet Dog48-49, fonds MarcelGriaule).

58. Registre de 1953 : 715 (fonds Marcel-Griaule).

59. Fiches «Binou » des 26 et 27 août 1948 (paquet Dog48, fonds Marcel-Griaule].

60. Fiches «Création » des 21 et 24 décembre 1948 (paquet Dog4848, fonds Marcel-Griaule). 
À travers cette explication transparaît une vision cryptologique de la société dogon et de sa culture matérielle. Relevant de codes symboliques plus ou moins secrets, les jeux ou les jouets "transcriraient" les mythes ou s'articuleraient à eux ${ }^{61}$, mais d'une manière cachée et détournée, grâce à différents subterfuges visuels. Dans sa variante cryptographique, cette même idée s'exprime avec force lors de l'analyse du tambour enfantin kunyu. Selon Griaule, la version originelle et oubliée de l'objet renfermait des dessins ésotériques invisibles de l'extérieur :

Ces jouets recouverts de peau de rat [...] étaient autrefois peints, intérieurement, de signes élémentaires connotant la formation des êtres et le début du peuplement de l'univers. La caisse semi oblongue est d'ailleurs une matrice ouverte projetant à l'aide de sons, dans les terres vides, les germes utiles et les volontés de croissance contenus dans les schémas aujourd'hui oubliés. Mais l'analyse profonde de ce tambour et des gesticulations enfantines révélerait que cette orchestration de fin d'été contient à elle seule la totalité de l'histoire du monde (Griaule 1952b : 21).

Fig 11 Figurine zoomorphe kouroumba collectée en 1939 lors de la mission LebaudyGriaule (musée du quai Branly).
Aux jeux dogon "terre à terre" tels qu'ils sont présentés en 1938, Griaule substitue donc, à partir de 1948, des jeux idéels, connectés aux étoiles ou aux mythes, et garants de la bonne marche de l'univers. Cette tendance culmine, neuf ans après la mort de Griaule, avec la publication en 1965 du livre intitulé Le Renard pâle. Proche collaboratrice de Griaule, avec qui elle signe l'ouvrage, Germaine Dieterlen a effectué une synthèse de leurs travaux antérieurs pour aboutir à un mythe dogon linéaire qu'elle ne cesse de relier à des objets courants, afin de donner à cette cosmogonie une assise matérielle et tangible. Au fil des pages, différents épisodes mythiques sont ainsi "rappelés ", "illustrés " ou «connotés " par le tourniquet gangala, les jouets en terre, les jeux de ficelle ou les différents tambours utilisés par les enfants (Griaule et Dieterlen 1965 : 193, 195, 244, 269, 452).

Mais, en fusionnant dans un même récit les différentes versions recueillies depuis 1948, Dieterlen en oublie parfois de faire la distinction entre les jeux réels, directement observables, et leur prototype présumé, entièrement et idéalement façonné par le mythe. En tant que "représentation de l'arche du Nommo ", le jeu walia est décrit comme une caisse en bois comportant " deux rangs de huit cupules » (ibid. : 452), ainsi qu'on peut le constater sur le dessin de la page suivante. À aucun moment il n'est précisé que ce jeu, tel qu'il a été observé par Griaule ${ }^{62}$ (et tel qu'il est observable aujourd'hui encore), compte douze cases et non seize. Plus troublant encore, le dessin d'illustration est un croquis de Griaule auquel on s'est contenté d'ajouter quatre cases de façon à obtenir les seize trous escomptés (Griaule 1938 : fig. 94B, 166). On arrive ainsi au paradoxe suivant : certains jeux, utilisés pour illustrer concrètement les projections du mythe dans la réalité, ne sont en fait que des objets chimériques créés à partir de ce mythe. Du reste, lorsque Dieterlen qualifie le walia à seize cases d'objet «réglementaire ${ }^{63}$ ", elle évoque de toute évidence sa conformité au mythe et non aux usages. Au terme des recherches menées par Griaule et Dieterlen, les jeux, d'abord associés à des pratiques ordinaires, sont désormais rivés aux mythes, et calqués sur eux. Mais s'agit-il encore de divertissements enfantins ou a-t-on déjà basculé vers des jeux intellectuels propres aux adultes? 
De 1952 à 1956, Griaule reproduit dans ses carnets de terrain de nombreux dessins tracés à sa demande par ses informateurs privilégiés pour éclairer, modéliser ou synthétiser leurs récits cosmogoniques. Il s'agit d’abord de simples «signes " soigneusement calligraphiés, mais plus les discours de ses interlocuteurs se complexifient et s'harmonisent, plus ces graphies «exemplaires " gagnent en précision, en taille, en couleur et en élégance, occupant pour finir une pleine page de cahier, à l'image des magnifiques croquis de génies d'eau pisciformes dessinés en $1955^{64}$. Présentés comme des figures de démonstration ou des signes ésotériques peints sur les façades des sanctuaires et sur les autels (ou sous eux), ces splendides dessins sont reproduits par centaines dans Le Renard pâle (1965), où ils sont de nouveau valorisés jusqu'à occuper une pleine page pour les plus beaux d'entre eux. Or, de par la finesse et la régularité de leurs traits, ces illustrations somptueuses, produites par (et pour) l'enquête, se rapprochent davantage d'une œuvre d'art que des figures au tracé grossier censées leur servir de modèle, en l'occurrence les peintures exécutées en bouillie de mil ou de $\mathrm{riz}^{65}$. Fasciné et admiratif, Griaule met d'ailleurs sous verre certains de ces dessins savants, qu'il estime pourtant indépendants de toute créativité individuelle. Ils forment selon lui un "corpus» de graphies invariables et ordonnées, autrement dit un système structuré de signes, à la frontière de l'art sacré et du langage symbolique ${ }^{66}$.

Tels qu'ils sont perçus par Griaule au début des années 1950, les dessins des «initiés » dogon ne sont ni de lointains reflets ni de plaisantes illustrations de la cosmogonie, contrairement aux jeux. Alliant beauté formelle et signification ésotérique, ils transcrivent secrètement le mythe tout en le dessinant avec art, ou au minimum avec soin, à l'opposé des graffiti enfantins, dépourvus de valeurs religieuse et esthétique. Les équivalences implicitement posées entre graphisme élaboré, signification complexe, savoir supérieur et codification extrême (propres aux «initiés"

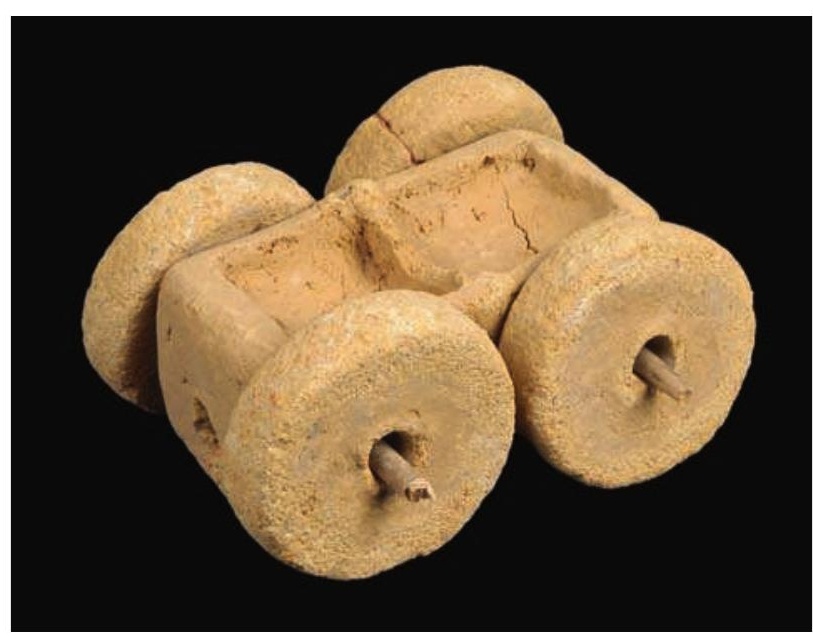
adultes) sont d'ailleurs l'envers logique des liens supposés entre grossièreté du dessin, sens élémentaire, connaissance de base et liberté expressive (propres aux enfants). En raison de ces associations, toute production graphique chargée de sens, qu'il soit symbolique ou mythologique, se doit d'être "propre» et élégante, par opposition aux gribouillis enfantins, et cette règle s'applique aussi bien à une figure «rituelle » qu'à un croquis de travail purement didactique. Si l'un écrit le mythe tandis que l'autre l'explique, ces deux types de dessins "sérieux" ont en effet le même objectif aux yeux de Griaule : donner forme à des mythes merveilleux sans trahir leur cohérence, leur profondeur et leur «fabuleuse » beauté.

Tel qu'il est présenté et construit par Griaule, le système graphique et cosmogonique des Dogon correspond à un double aboutissement : il est la cime commune de l'art, du savoir et de la religion, mais il est également le résultat final d'une longue «initiation » impliquant aussi bien les Dogon que les ethnographes. Certains informateurs, comme Amadigné et Koguèm, ont même progressé vers ce sommet avec Griaule, depuis l'enfance et à partir des jeux. Dans cette perspective ascendante, on peut d'ailleurs se demander si les recherches de Griaule et de ses collaborateurs ne trouvent pas leur limite dès qu'elles atteignent leur objectif, c'est-à-dire dès qu'elles parviennent à ce savoir sommital. Mais, en dépit
Fig 12 Jouet dogon représentant une automobile (mission Sahara-Soudan, 1935. Musée du quai Branly).

\section{- -}

64. Voir registre de septembre 1955 : 973 bis, 974 bis, 978 bis (fonds Marcel-Griaule). Tous ces dessins se trouvent reproduits en pleine page et parfois en couleurs dans Le Renard pâle (Griaule et Dieterlen 1965 : 158159, 161, 445).

65. Dans Le Renard pâle, les très rares reproductions de peintures originales se distinguent justement par leurs contours grossiers (ibid. : 453, fig. 158).

66. Griaule 1952 : 541 . À partir de 1952, Griaule commence d'ailleurs à classer et numéroter les signes de ce corpus présumé. 


\section{- $\bullet$}

67. Griaule 1983 [1937] : 9.

À propos des jeux, Griaule écrit notamment : «malgré le machinisme et le progrès de la raison pure, nos enfants vivront toujours dans le merveilleux 》 (Griaule $1938: 27$ ). des déclarations d'intention, le but personnel de Griaule est peut-être moins de gravir doctement toutes les marches de la connaissance dogon que d'explorer avec enthousiasme un territoire vierge et merveilleux qui serait la version "élevée » et ordonnée de l'imaginaire des enfants ou de leurs activités ludiques. Du reste, si Griaule oppose les marges enfantines et les confins mythologiques, il les place en définitive dans un même continuum en situant les jeux dans le prolongement des mythes et à la base du savoir. Et il les regarde tous deux comme des mondes originels, savoureux et préservés, restés à l'écart d'un « progrès mécanique » jugé abominable et insipide ${ }^{67}$.

CNRS, Centre d'études des mondes africains (CEMAf) jolly@ivry.cnrs.fr

mots clés / keywords : Griaule // Griaule $\cdot$ enfant // child • jeu // game $\cdot$ mythe // myth • dessin // drawing • Dogon // Dogon • Éthiopie // Ethiopia.

\section{Bibliographie}

\section{ANONYME \\ 1933 «Le musée d'ethnographie continue inlassablement de nous instruire et de nous émerveiller », Mercure de France, $1^{\text {er }}$ septembre : 448-452. \\ BARRUCAND, Lucienne \\ 1935 « M. Marcel Griaule à la salle Pierre Bordes : Les Dogons du Niger », Dépêche algérienne, 10 avril.}

\section{Bataille, Georges}

1930 «L'art primitif », Documents $7:$ 389-39?.

\section{Bosc-Tiessé, Claire}

2002 « Musée de l'homme : la décoration de carreaux de faïence hollandais d'une église royale éthiopienne au xvII siècle (Qwesqwam, Gondar] », Revue du Louvre 3, juin : 54-59.

Bosc-Tiessé, Claire, et Wion, Anaïs

2005 Peintures sacrées d'Éthiopie. Collection de la Mission Dakar-Djibouti. Saint-Maur-des-Fossés, Éditions Sépia.

\section{Chastenet, Jacques}

1957 «Marcel Griaule, l'homme », Marcel Griaule, conseiller de l'Union française. Paris, Nouvelles Éditions latines: 20-22.

\section{CoHen, Marcel}

1911 « Jeux abyssins 》, Journal asiatique XVIII, novembre-décembre : 463-49?.
FORTOLIS, L.

1931 «La mission Griaule s'achemine vers Tombouctou », La Liberté, 4 octobre.

\section{FOUCART, George}

1919 Questionnaire préliminaire d'ethnologie africaine. Le Caire, Imprimerie de l'Institut français d'archéologie orientale.

\section{Gordon, Hélène}

1935 « Chez les hommes des cavernes de l'Afrique noire. Dans l'antre des démons buveurs de sang », L'Intransigeant, (I) $?$ mai : 1-2, (II) 8 mai : 1-2, (III) 9 mai : 1-2 ; (VIII) 14 mai : $1-2$.

\section{Griaule, Marcel}

1929 « Jeux abyssins », Documents $6: 332$.

1930 « Joujou », Documents 6 : 367-368.

1930b « En Abyssinie », La Géographie LIV, novembre-décembre : 359-370.

1931 «Buts et méthode de la prochaine mission Dakar-Djibouti », conférence prononcée au MET le soir de l'inauguration de l'exposition [du matériel de] Dakar-Djibouti (fonds Marcel-Griaule, Labethno, Nanterre) 1932 «Mission Dakar-Djibouti », Rapport général (mai 1931 - mai 1932), Journal de la Société des africanistes $\|$ : 113-122.

1932 b « Mission Dakar-Djibouti », Rapport général (juin à novembre 1932), Journal de la Société des africanistes 11 : 229-236.
1933 «Introduction méthodologique 》, Minotaure 2 : 7-12.

1933b Silhouettes et graffiti abyssins. Paris, Larose.

$1933 c$ « Le chasseur du 20 octobre », Minotaure 2 : 31-34

$1933 d$ « Peintures abyssines », Minotaure 2 : 83-88.

1934 «Le jeu de mail en Abyssinie », Bulletin du Musée d'ethnographie du Trocadéro ?, janvier-juin : 11-15.

1934b Les Flambeurs d'hommes. Paris, Calmann-Lévy.

1934c «La mission Dakar-Djibouti », Bulletin de l'Association française pour l'avancement de la science, décembre : 413-420.

1935 Jeux et divertissements abyssins. Paris, Librairie Ernest Leroux.

1938 Jeux dogons. Paris, Institut d'ethnologie. 1943 « Cinq missions ethnographiques en Afrique tropicale », Comptes rendus des séances de l'Académie des sciences coloniales IX (séances des 3 et 17 décembre) : 680-688. 1943b Les Sao légendaires. Paris, Gallimard. 1948 Dieu d'eau. Entretiens avec Ogotemmêli. Paris, Les Éditions du Chêne.

1948b «L'arche du monde chez les populations nigériennes », Journal de la Société des africanistes XVIII : 117-128.

1952 «L'enquête orale en ethnologie », 
Revue philosophique de la France et de l'étranger CXLII, octobre-décembre : 537-553.

$1952 b$ « Réflexions sur des symboles

soudanais 》, Cahiers internationaux de sociologie XII : 8-30.

1952 c « Le savoir des Dogon », Journal de la Société des africanistes XXII (1-2) : 27-42.

1953 «Le problème de la culture noire », L'Originalité des cultures, son rôle dans la compréhension internationale. Paris, Unesco : 374-401.

1955 «Rôle du silure Clarias Senegalensis dans la procréation au Soudan français », Afrikanistische Studien, vol. 26 : 299-311.

1957 Méthode de l'ethnographie. Paris, PUF. $1957 \mathrm{~b}$ «Le trafic des esclaves », Marcel Griaule, conseiller de l'Union française. Paris, Nouvelles Éditions latines : 150-154.

1983 [1937] Préface au livre de Joseph-Marie Lo Duca La Sphère de platine. Paris, Denoël : 8-9.

Griaule, Marcel, et Dieterlen, Germaine

1965 Le Renard pâle. Paris, Institut d'ethnologie.

Griaule, Marcel, et Ligers, Ziedonis

1955 « Le bulu, jeu bozo », Journal de la

Société des africanistes XXV (1-2) : 35-37.

INSTRUCTIONS SOMMAIRES POUR LES COLLECTEURS D'OBJETS ETHNOGRAPHIQUES

1931 Paris, Musée d'ethnographie et Mission scientifique Dakar-Djibouti.

\section{JAMIN, Jean}

1982 « Objets trouvés des paradis perdus

[à propos de la Mission Dakar-Djibouti] », in

J. Hainard et R. Kaehr (éd.), Collection passion.

Neuchâtel, musée d'Ethnographie : 69-100.

\section{Jolly, Éric}

2001-2002, « Du fichier ethnographique au fichier informatique. Le fonds Marcel Griaule : le classement des notes de terrain 》, Gradhiva 30-31 : 81-103.

\section{LABOURET, Henri}

1933 Plan de monographie régionale.

Paris, Larose.

\section{Lebeuf, Jean-Paul}

1941 «Poupées et bâtons fali », Journal de la Société des africanistes XI (1-2) : 226-22?.

1945 Quand l'or était vivant. Aventures au Tchad. Paris, J. Susse.

1983 «Poupées d'Afrique parmi tant d'autres », Poupée-jouet, poupée reflet. Paris, Muséum national d'histoire naturelle : 97-99.

1991 « Jeux éphémères 》, Journal des africanistes 61 (1) : 139-144.

\section{Leiris, Michel}

1933 «L'exposition de la Mission Dakar-Djibouti (1931-1933) », La Terre et la vie ?, juillet : 431-432. 1981 [1934], L'Afrique fantôme. Paris, Gallimard.

1996 Miroir de l'Afrique. Paris, Gallimard (édition établie, présentée et annotée par Jean Jamin].

\author{
Luouet, Georges-Henri \\ 1930 L'Art primitif. Paris, G. Doin \& Cie. \\ LuTTEN, Éric \\ 1933 « Poupées d'Afrique occidentale \\ (recueillies par la Mission Dakar-Djibouti) », \\ Bulletin du Musée d'ethnographie du Trocadéro \\ 5, janvier : 8-19. \\ 1934 « Les enfants noirs ont aussi des \\ poupées », Le Monde colonial illustré 129, \\ mai : 79.
}

\section{Mauss, Marcel}

1947 Manuel d'ethnographie. Paris, Payot.

\section{Mauss, Marcel, et Griaule, Marcel}

1933 «Introduction », in M. Griaule, Silhouettes et graffiti abyssins. Paris, Larose : 5-6.

\section{Notes AND Queries on Anthropology}

1929 Londres, The Royal Anthropological Institute.

\section{SAвот, Philippe}

2003 «Primitivisme et surréalisme : une "synthèse" impossible ? », Methodos 3 : 113-136.

\section{Archives ethnographiques}

Fonds Dakar-Djibouti (1931-1933), bibliothèque Éric-de-Dampierre, MAE, Université de Paris Ouest, Nanterre, La Défense.

Fonds Marcel-Griaule (1926-1956), bibliothèque Éric-de-Dampierre, MAE, Université de Paris Ouest, Nanterre, La Défense.

\section{Résumé / Abstract}

Éric Jolly, Des jeux aux mythes : le parcours ethnographique de Marcel Griaule - Lors de ses missions africaines, de 1928 à 1956, Marcel Griaule travaille d'abord sur les jeux et les dessins des enfants avant de centrer ses recherches sur le savoir mythologique et graphique des vieillards ou des «initiés 》 dogon. Cet article analyse les raisons de cette progression thématique des activités ludiques vers les systèmes cosmogoniques, des dessins naïfs vers les signes ésotériques, et des enfants innocents vers les adultes «savants». Explorateur de territoires vierges ou préservés, Marcel Griaule construit son parcours ethnographique comme une patiente ascension depuis le monde originel de l'enfance jusqu'aux connaissances secrètes et figées censées coiffer l'ensemble de la société. L'imaginaire des enfants et les mythes des adultes sont en effet les deux pôles opposés (et hiérarchisés] d'un même univers merveilleux qui l'attire et le fascine.
Éric Jolly, From games to myths: the ethnographic career of Marcel Griaule - During his African expeditions, from 1928 to 1956, Marcel Griaule worked first of all on children's games and drawings before focusing his research on the mythological and graphic knowledge of old people or Dogon 'initiates'. This article analyses the reasons for this thematic progression from ludic activities to cosmogonic systems, from naïve drawings to esoteric signs, and from innocent children to 'erudite' adults. As an explorer of virgin or preserved territories, Marcel Griaule constructed his ethnographic career as a patient ascent from the original world of childhood to the secret and congealed knowledge that capped the whole of society. The childhood imaginary and adult myths are indeed the two opposed (and hierarchical) poles of the same marvellous universe which attracted and fascinated him. 